\title{
Study of Galaxies in the Lynx-Cancer Void. IV. Photometric Properties
}

\author{
Yu. A. Perepelitsyna, ${ }^{1, *}$ S. A. Pustilnik, ${ }^{1,{ }^{* *}}$ and A. Yu. Kniazev ${ }^{2,3,4,{ }^{* * *}}$ \\ ${ }^{1}$ Special Astrophysical Observatory, Russian Academy of Sciences, Nizhnii Arkhyz, 369167 Russia \\ ${ }^{2}$ South African Astronomical Observatory, Cape Town, 7935 South Africa \\ ${ }^{3}$ Southern African Large Telescope, Cape Town, 7935 South Africa \\ ${ }^{4}$ Lomonosov Moscow State University, Moscow, 119991 Russia
}

(Received December 13, 2013; Revised May 14, 2014)

We present the results of a photometric study of 85 objects from the updated sample of galaxies residing in the nearby Lynx-Cancer void. We perform our photometry on $u, g, r$, and $i$-band images of the Sloan Digital Sky Survey. We determine model-independent galaxy parameters such as the integrated magnitudes and colors, effective radii and the corresponding surface brightness values, optical radii and Holmberg radii. We analyze the radial surface brightness profiles to determine the central brightness values and scale lengths of the model disks. We analyze the colors of the outer parts of the galaxies and compare them with model evolutionary tracks computed using the PEGASE 2 software package. This allowed us to estimate the time $T_{\mathrm{SF}}$ elapsed since the onset of star formation, which turned out to be on the order of the cosmological time $T_{0}$ for the overwhelming majority of the galaxies studied. However, for 13 galaxies of the sample the time $T_{\mathrm{SF}}$ does not exceed $T_{0} / 2 \sim 7 \mathrm{Gyr}$, and for 7 of them $T_{\mathrm{SF}} \lesssim 3.5$ Gyr. The latter are mostly unevolved objects dominated by low-luminosity galaxies with $M_{B}>-13.2$. We use the integrated magnitudes and colors to estimate the stellar masses of the galaxies. We estimate the parameter $\mathfrak{M}(\mathrm{HI}) / L_{B}$ and the gas mass fractions for void galaxies with known H I-line fluxes. A small subgroup (about $10 \%$ ) of the gas-richest void galaxies with $\mathfrak{M}(\mathrm{HI}) / L_{B} \gtrsim 2.5$ has gas mass fractions that reach 94-99\%. The outer regions of many of these galaxies show atypically blue colors. To test various statistical differences between void galaxies and galaxies from the samples selected using more general criteria, we compare some of the parameters of void galaxies with similar data for the sample of 195 galaxies from the Equatorial Survey (ES) based on a part of the HIPASS blind HI survey. The compared samples have similar properties in the common luminosity range $-18.5<M_{g}<-13.5$. The faintest void galaxies differ appreciably from the ES survey galaxies. However, the ES survey also contains about $7 \%$ of the so-called "inchoate" galaxies with high $\mathfrak{M}(\mathrm{HI}) / L_{B}$ ratios, most of which are located far from massive neighbors and are probably analogs of void galaxies.

Keywords: galaxies: photometry-galaxies: fundamental parameters-galaxies: evolution 


\section{INTRODUCTION}

Voids in the large-scale distribution of galaxies were discovered more than 30 years ago (e.g., [1, 2]). Observationally they are commonly defined as regions with no galaxies of normal and high luminosity: $M_{B} \geq-20$, which corresponds to the break in the galaxy luminosity function (e.g., [3]). Voids occupy more than half of the volume of the present-day Universe. Note that the number of galaxies in the voids does not exceed $20 \%$ of the total number of catalogued galaxies, and this fraction is indicative of a significantly lower mass density inside these structures. In numerical models of the evolution of matter in the hot Universe with dark matter $(\mathrm{DM})$, voids arise as natural structures and, on the whole, resemble the observed voids rather closely. However, the number of galaxies observed in voids is several times smaller than the predicted number of gravitationally bound DM haloes. The cause of this phenomenon, which was formulated in different forms by de Lapparent [4], Peebles [5], and Tikhonov and Klypin [6], remains unclear.

It is hoped that further improvement of models of the formation and evolution of galaxies will reduce the gap between the number of DM haloes and real galaxies. The above phenomenon may be caused, among other things, by the

\footnotetext{
*** E-mail:akniazev@saao.ac.za
}

higher fraction of low surface brightness galaxies (LSBD) in the voids, because such objects are more difficult to detect and identify. More detailed and extensive studies of the properties of galaxies in the voids are needed to understand the situation with such objects. Most of the authors working in this direction (e.g., [7-9]) studied large distant voids and, because of natural observational selection, dealt only with the brightest void galaxies $\left(M_{r} \lesssim-16.5\right)$. No substantial differences have been found between these galaxies and similar galaxies in denser structures, except that the former have somewhat bluer colors. In their recent paper Hoyle et al. [10] studied the photometric properties of almost 90000 galaxies in about 1000 voids by analyzing their SDSS images. They similarly found that dwarfs of all types in voids are systematically bluer compared to their analogs in denser environments.

One would expect, based on general considerations and simulations, that the effects of the environment on the evolution should be most significant for the least massive galaxies (e.g., [11]). The currently available results suggest that the following factors should influence the evolutionary status of low-mass galaxies in the voids.

(1) An appreciably delayed formation of gravitationally bound DM halos with masses typical of dwarf galaxies in low-density large-scale structures (voids) [12] and slow evolution of (a part of) void galaxies.

(2) Voids are filled mostly with low-mass galax- 
ies. Therefore, because of the well-known correlation between luminosity and surface brightness (e.g., [13]), their population contains a higher fraction of LSB galaxies. Numerical simulations of the interaction of low- and normal(high-, HSB) surface brightness disk galaxies [14] in the case of their encounters without mergers show that the final response differs strongly for the two types of objects. HSB galaxies respond to such interactions by developing a bar and a mature burst of star formation, whereas the response in LSB galaxies is much weaker with the star-formation rate increasing only slightly. The number of collisions in voids during the lifetime of a galaxy is several times smaller than in the average-density regions, and therefore one would expect an appreciable fraction of void galaxies to have never been affected by "significant" interactions. However, these general considerations should be validated by numerical simulations taking into account many subtle details of the formation and evolution of galaxies.

(3) Kreckel et al. [11] analyzed the results of such a simulation of the evolution of galaxies in voids and found evidence suggesting that these galaxies differ from the galaxies in denser regions, but this is only true for the lowest-mass objects in their modeled mass range (which corresponds to luminosities $M_{r}$ ranging from $-12^{\mathrm{m}}$ to $\left.-16^{\mathrm{m}}\right)$.

It follows from the above that one must search for the possible evolutionary peculiarities in the least massive void galaxies by studying sufficiently close objects.

The first deep sample of 79 galaxies (down to $\left.M_{B} \sim-12\right)$ in the nearby Lynx-Cancer void was presented by Pustilnik and Teplyakova [15]. The results of their study suggest that the effect of low-density environment indeed exists for such low-mass galaxies, and that it manifests itself in the slower rate of evolution. According to the conclusions of Pustilnik et al. [16], void galaxies have systematically lower metallicities (on the average, by 30\%) compared to the galaxies in denser environments. Furthermore, a small but quite significant (about 10\%) fraction of void galaxies have peculiar properties typical of evolutionarily young (unevolved) objects $[17,18]$. We should also mention the socalled Void Galaxy Survey (VGS, [19]) carried out to study the H I structure and optical properties of about 60 SDSS galaxies residing near the centers of large but relatively distant (approximately $80 \mathrm{Mpc}$ ) voids. Only for some of the closest voids of this sample, the luminosities of the galaxies studied are more or less close to the median luminosity of the sample in the Lynx-Cancer void. It is among such galaxies that Kreckel et al. [19] found three very gas-rich objects.

In this paper we report the results of our photometric study of the galaxies in the LynxCancer void based on their $u, g, r, i$-band images from the SDSS DR7 (Sloan Digital Sky Survey Data Release 7) database [20]. The aim of our 
photometric analysis is to determine the basic parameters of the sample galaxies in order to study the statistical properties of void galaxies and compare the results with those obtained for other samples of similar galaxies in denser environments or samples based on other criteria. The integrated magnitudes and color indices of the galaxies can be used to estimate their stellar masses. Furthermore, the colors of the outer regions of the galaxies, which usually bear no traces of recent or ongoing star formation, are compared to model evolutionary tracks and used as age indicators for the oldest (visible) stellar population. One of the aims of this study is to estimate these ages.

\section{UPDATED GALAXY SAMPLE IN THE LYNX-CANCER VOID}

A detailed description of the original sample of galaxies in the Lynx-Cancer void can be found in our earlier paper [15], where we present a list of 79 galaxies residing in this void-a simplyconnected domain containing no galaxies of normal and high luminosity (here $M_{B}<-19.0$ ). The void is described by a sphere with a radius of $8 \mathrm{Mpc}$ together with the adjoining regions. The void galaxies satisfy the condition of sufficient separation (more than $2 \mathrm{Mpc}$ ) from galaxies brighter than $M_{B}=-19.0$. We discuss the completeness of the sample in the same paper. As of now, more than 20 new galaxies of the void have been found. Some of them were discovered as a result of a program of the search for new LSB dwarf galaxies in this void, and the remaining ones - as a result of new surveys (mostly the ALFALFA survey [24]) and a detailed analysis of the data for the already known galaxies in this sky area.

We are preparing the updated sample of galaxies of the Lynx-Cancer void for publication. The new version includes 101 galaxies, 16 of which lie outside the sky area covered by SDSS fields. We limit our analysis to 85 galaxies listed in Table 1. We base our study on the updated sample in order to study the photometric properties of void galaxies with maximum completeness.

\section{METHODS OF PHOTOMETRIC REDUCTION}

The SDSS survey [25] offers high-quality calibrated images taken in the $u, g, r, i, z$ bands. Therefore primary reduction has to include only one extra step-thorough subtraction of the sky background. We performed this procedure in the ESO-MIDAS environment using the aip package as described in detail in the paper by Kniazev et al. [26]. The object under study is masked and the sky background is approximated by a two-dimensional polynomial. The resulting sky background model is then subtracted from the image. The sky background in the masked region is interpolated from the surrounding region. Ring aperture photometry is then performed to 
measure the flux inside the masked region for the $u, g, r, i$-band images with subtracted background. The inferred fluxes are then converted into integrated magnitudes of the galaxies. To convert the instrumental fluxes into magnitudes, we use a set of photometric coefficients for each field of the SDSS database.

We determine the following modelindependent parameters for all galaxies: integrated $u, g, r, i$-band magnitudes, integrated $(u-g),(g-r),(r-i)$ colors, and integrated $B_{\text {tot }}$ magnitudes converted from the $g$ - and $r$-band magnitudes by the formulas proposed by Lupton [27]. After creation of the surface brightness profile, we estimate for each object the radii $R_{50}$ and $R_{90}$ containing $50 \%$ and $90 \%$ of the $u, g, r, i$-band fluxes of the galaxy. In addition, we estimate the effective surface brightness inside the $R_{50}$ radius, the observed minor-to-major semiaxis ratio $b / a$, the "optical" and Holmberg radii [28] (at the $\mu(B)=25.0$ and $26.5 / \square^{\prime \prime}$ surface brightness levels respectively). We multiply all the effective radii by a factor of $\sqrt{a / b}$.

Fitting an exponential or Sersic [29] law to the radial surface brightness profiles allowed us to determine the model parameters $\mu_{0}$ of the central $u, g, r, i$-band surface brightness. We then compute the $B$-band central brightness $\mu_{0}(B)$ from $\mu_{0}(g)$ and $\mu_{0}(r)$ by Lupton's formulas [27]. We also estimate the $u, g, r, i$, and $B$-band $\left(\mu_{0, c, i}(B)\right)$ central surface brightness values corrected for the inclination of the galaxy disk to the line of sight and the foreground extinction in our Galaxy in accordance with [30]. We compute the inclination correction by the formula $\delta \mu=-2.5 \log (\cos i)$, where $i$ is the angle between the disk plane and the line of sight. For objects with inclinations $i>70^{\circ}$, we compute the corresponding correction by the formula $\delta \mu=2.5 \log \left(\alpha / \alpha_{z}\right)\left(1-\exp \left(-\tan (i) \alpha / \alpha_{z}\right)\right)[31]$, where we set the $\alpha / \alpha_{z}$ scale length ratio equal to 5 [32]. We compute both corrections assuming negligible internal extinction in the galaxies considered. We compute the angle $i$ by the standard formula $\cos ^{2} i=\left(p^{2}-q^{2}\right) /\left(1-q^{2}\right)$, where $p=b / a$ and $q$ are the observed axial ratio and the axial ratio of the real disk respectively. We set $q=0.2$ for relatively bright late-type galaxies and $q=0.4$ for low-luminosity objects with $M_{B}>-14.8$ in accordance with the results of Roychowdhury et al. [33]. We also estimate the (characteristic) disk scale length $\alpha$ for the $u, g, r, i$-band images and for the Sersic exponent $n$.

\subsection{Determination of the Ages} of the Old Stellar Population

We estimate the colors of the outer parts of the galaxies by performing additional photometry inside a set of small circular apertures (with the radii of about $4^{\prime \prime}$ ) at the periphery of the galaxies excluding small higher-luminosity areas. We then sum up the flux in these apertures and convert it into magnitudes and colors. For the 


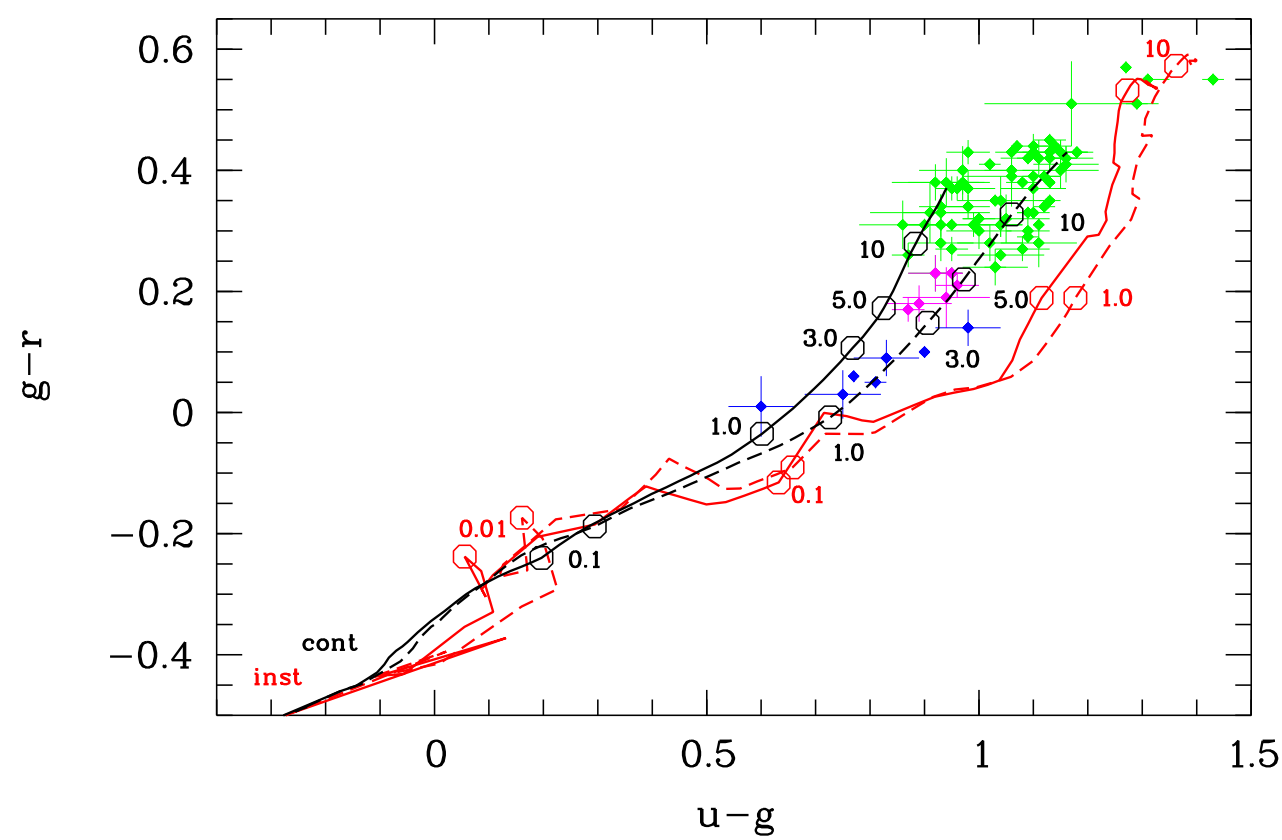

Figure 1. The $(u-g)-(g-r)$ color-color diagram of the outer parts of 85 galaxies of our sample of the Lynx-Cancer void compared to PEGASE2 evolutionary tracks for two extreme cases of the star-formation law. Instantaneous (inst) SF for two initial mass functions (IMF): Salpeter (the red solid track) and Kroupa (the red dashed track). Continuous (const) star formation for the same two IMFs: Salpeter (the black solid track) and Kroupa (the black dashed track). The green, pink, and blue circles show the colors of the outer parts of the galaxies corresponding to the ages $T \sim 10-13$ Gyr, $T \sim 4-6.5$ Gyr, and $T \sim 1-3.5$ Gyr respectively. The numbers near each track indicate the time in Gyr elapsed since the onset of star formation.

objects with uncertain positions of the HII regions, we use the $\mathrm{H} \alpha$ images taken with the 6-m telescope of the Special Astrophysical Observatory. We estimate the time elapsed since the onset of an epoch or a "short episode" of star formation, which under certain assumptions can be used as an age estimate for the oldest visible stars, by comparing the colors of the peripheral regions of galaxies with model evolutionary tracks computed using the PEGASE2 software package [21].

When selecting the peripheral areas to determine the colors of the oldest population, we choose the areas located far from the center but where the fluxes could still be measured with reasonable accuracy. As a result, the surface brightness levels of the considered peripheral areas vary substantially for galaxies with different surface brightness and/or characteristic size ranging from $\mathrm{SB}_{B}=24.5 / \square^{\prime \prime}$ to $26 . \mathrm{m} 9 / \square^{\prime \prime}$ with a median value of $\mathrm{SB}_{B}($ median $)=25^{\mathrm{m}} \cdot 3 / \square^{\prime \prime}$. We convert the $\mathrm{SB}_{g}$ values, actually measured in the radial surface brightness profiles, into $\mathrm{SB}_{B}$ parameters adopting the color index $B-g=0.4$ typical of void galaxies. 


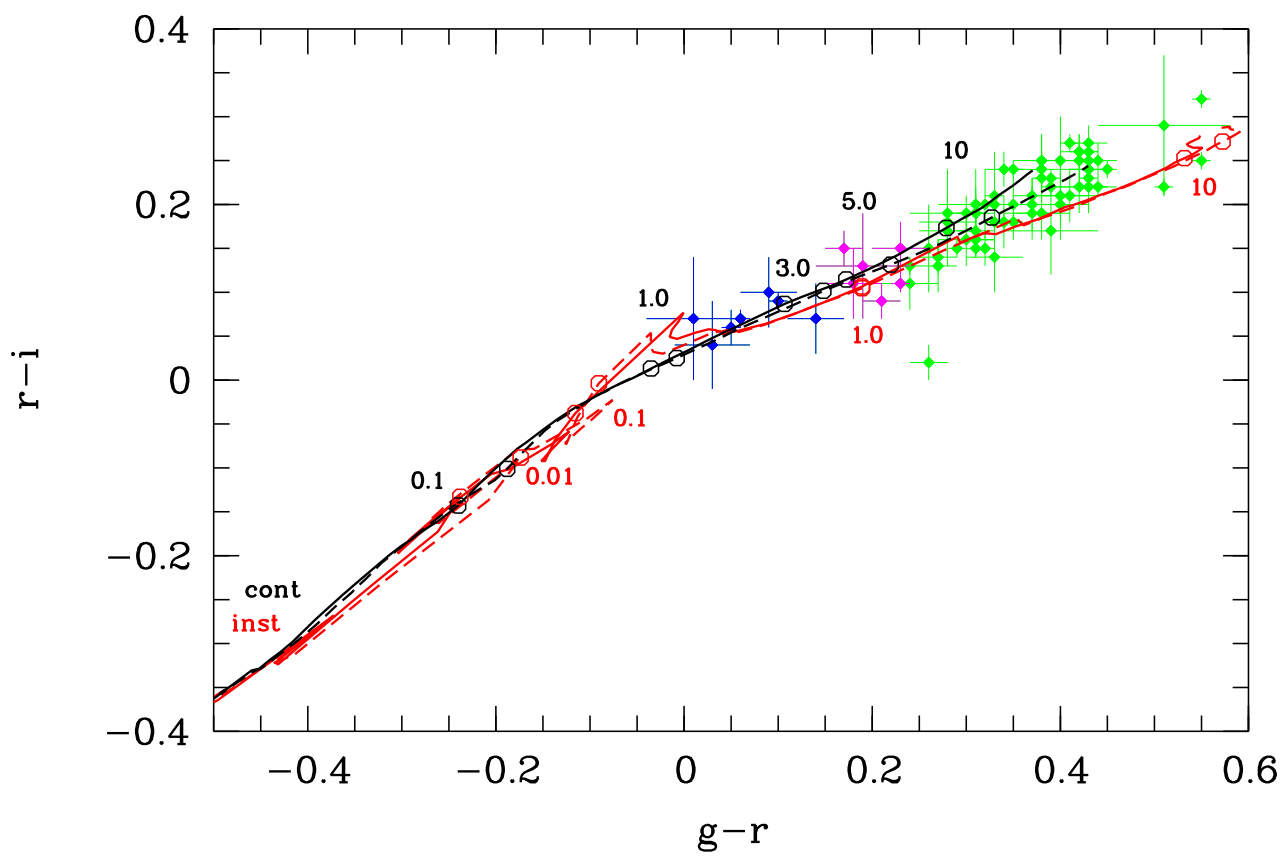

Figure 2. The $(g-r)-(r-i)$ color-color diagram of the outer parts of 85 galaxies in the Lynx-Cancer void compared to PEGASE2 evolutionary tracks for two extreme cases of the star formation law (same designations as in Fig. 1).

The PEGASE2 software package is designed for computing models of the photometric evolution of galaxies. The main parameters of the models are: the initial mass function $\varphi(\mathfrak{M})=\mathfrak{M}^{-(1+x)}$, the star-formation law $\operatorname{SFR}(t)$, and stellar metallicity $Z$. We use for our analysis the initial mass functions (IMF) of Salpeter [34] and Kroupa et al. [35], which best describe the observed distributions of stellar masses in the nearby galaxies. We use two extreme scenarios for the star-formation law: an instantaneous episode-a burst - of star formation (inst) and star formation at a constant rate (const). The tracks of all other non-exotic scenarios lie between these two extreme cases. For any fixed time since the onset of star formation, the const scenario track produces the bluest colors, because the fraction of massive blue stars for this track decreases at a slower rate than the corresponding fraction for the inst scenario track. In the domains of the ugr diagram where the const and inst tracks run sufficiently close to each other (see Fig. 1), the time elapsed since the onset of star formation and, correspondingly, the ages of the oldest stars are about five times greater for the const track than the corresponding quantities for the inst tracks. As a result, in the cases of uncertain estimates, the const tracks yield robust upper limits for the estimated ages of the oldest stars, which therefore can be used as the upper estimates for the ages of the visible extended old population. We show all our inferred colors of the outer regions of the 
program galaxies in the ugr and gri two-color diagrams, where we compare these colors with the model tracks described above (Figs. 1 and 2).

When comparing the observed colors of the galaxies with those of model tracks, we choose the stellar metallicity for the computed tracks from a discrete set of values offered by PEGASE2 adopting the one that is the closest to the measured gas metallicity for a particular galaxy. In most cases we use one of the following three metallicity values: $Z=0.004, Z=0.002$, or $Z=0.001$.

The filter transmission curves used to compute the PEGASE2 evolutionary tracks slightly differ from the transmission curves of the $u, g, r, i$ filters used in actual SDSS observations. We use the formulas from Tucker et al. [36] to eliminate the resulting small biases.

\subsection{Gas Mass Fraction}

The total hydrogen mass $\mathfrak{M}(\mathrm{HI})$ for the galaxies of our sample with measured integrated 21-cm HI line fluxes $F(\mathrm{HI})$ can be estimated using the formula for the optically thin layer: $\mathfrak{M}(\mathrm{HI})=2.36 \times F(\mathrm{HI}) D^{2} 10^{5}$ [37], where $D$ and $F(\mathrm{HI})$ are the distance to the galaxy in $\mathrm{Mpc}$ and flux in $\mathrm{Jy} \mathrm{km} \mathrm{s}^{-1}$ respectively. One must also take into account the helium contribution $(0.33$ of $\mathfrak{M}(\mathrm{HI}))$ to estimate the total gas mass $\mathfrak{M}_{\text {gas }}$. The contribution of the mass of molecular gas in dwarf galaxies can be neglected in the first approximation. For about half of the galaxies, we adopt the $F(\mathrm{HI})$ data from the literature and determine the corresponding fluxes for the remaining galaxies from observations performed with the NRT radio telescope.

The photometry obtained as a result of this study can be used to estimate the total stellar masses. Given the gas mass and that of the stars, an evolutionary parameter-the gas mass fraction - can be determined. The total stellar mass $\mathfrak{M}_{*}$ is commonly estimated from the integrated photometry of a galaxy in one of the broadband filters $\lambda$ using the corresponding parameter $\Upsilon_{\lambda}=\mathfrak{M}_{*} / L_{\lambda}$-the mass-to-luminosity ratio for the stellar population, which depends on the color of the stellar continuum. We use for our estimates of $\mathfrak{M}_{*}$ the mass-to-luminosity ratio $\Upsilon$ and its dependence on the color indices as derived by Zibetti et al. [38]. More precisely, we use the ratio $\Upsilon_{g}(g-i)$ and $g$-band luminosity, because this combination yields the most robust results in the optical part of the spectrum. In our opinion, the mass-to-luminosity ratio $\Upsilon$ from [38] takes most adequately into account the complex history of star formation in low-mass galaxies including the recent episodes.

We use this stellar mass estimate $\mathfrak{M}_{*}$ to compute the baryonic mass $\mathfrak{M}_{\text {bary }}=\mathfrak{M}_{*}+\mathfrak{M}_{\text {gas }}$ of the galaxy and then the gas mass fraction $f_{\text {gas }}$, i.e., the ratio of the total gas mass to the total baryonic mass. To compare our results with those obtained for other samples, we also compute the model-independent parameter $\mathfrak{M}(\mathrm{HI}) / L_{B}$ which is the ratio of the hydrogen 
mass to $B$-band luminosity in solar units; this parameter is often used in less detailed studies. Here $L_{B}$ is determined from the $B_{\text {tot }}$ magnitude computed using Lupton's formulas [27] from our independent $g$ and $r$-band photometry. This parameter provides a coarse estimate of the gas mass fraction for galaxies with no color information available.

\section{RESULTS}

Table 1 lists the basic parameters for our sample of 85 galaxies of the Lynx-Cancer void. The columns in the table give: (1) the name of the galaxy; (2) the name of the object in the short IAU format; (3) type of the galaxy either adopted from [15] (for the objects from the original sample), or NED, ${ }^{1}$ or, if such information is unavailable in NED, according to our own estimate; (4) heliocentric radial velocity $V_{\text {hel }}$ in $\mathrm{km} \mathrm{s}^{-1}$; (5) distance to the object in Mpc (estimated as in [15], i.e., corrected for the large negative peculiar velocity in the region considered); (6) Galactic $B$-band extinction $A_{B}$ adopted from [30]; $(7,8)$ the total apparent (uncorrected for Galactic extinction) magnitudes $B_{\text {tot }}$ and the absolute (extinction-corrected) magnitudes $M_{B, 0}$ derived from our independent photometry by Lupton's formulas [27] and used in the subsequent statistical analysis; (9) total apparent $B_{\text {tot }}$ magnitudes adopted from NED (for comparison with

\footnotetext{
${ }^{1}$ NASA/IPAC Extragalactic Database, http://ned.ipac.caltech.edu
}

our magnitude estimates); (10) hydrogen mass $\mathfrak{M}(\mathrm{HI})$ in the units of $10^{7} M_{\odot}$ (according to the published data and the results of observations made with the NRT radio telescope); (11) hydrogen mass to $B$-band luminosity ratio $\mathfrak{M}(\mathrm{HI}) / L_{B}$ in solar units; (12) stellar mass $\mathfrak{M}_{*}$ in the units of $10^{7} M_{\odot} ;(13)$ gas mass fraction $f_{\text {gas }} ;(14)$ oxygen abundance $12+\log (\mathrm{O} / \mathrm{H})$; (15) the observed axial ratio $b / a$.

Model-independent parameters derived from photometry and SDSS image analysis are given in Tab. 2 and 3. In columns of Tab. 2 the following parameters are presented: (2),(3) the radii containing $50 \%$ and $90 \%$ of the $g$-band flux of the galaxy; (4) effective $g$-band surface brightness determined as the average surface brightness inside the $R_{50}(g)$ radius; (5),(6) $g$-band "optical" radius (at the $25 . \mathrm{m} 0 / \square^{\prime \prime}$ isophote level) in $\operatorname{arcsec}\left(R^{25}(g)\right)$ and kpc $\left(a_{25}\right)$; $(7) g$-band magnitude inside the "optical" radius; (8),(9) radius at the $26.5 / \square^{\prime \prime}$ isophote level (the Holmberg radius) [28] and the $g$-band magnitude inside this radius; (10),(11) optical size computed as $b=R^{25}(g) \sqrt{b / a}$ and $a=R^{25}(g) \sqrt{a / b}$ in arcsec and kpc respectively. In columns of Tab. 3 the following parameters are oresented: (2) integrated $g$-band magnitude; (3),(4),(5) Galactic extinction-corrected integrated colors $(u-g)$, $(g-r),(r-i) ;(6),(7),(8)$ the colors of outer parts $(u-g),(g-r),(r-i)$.

Fitting the photometric profiles to an exponential disk or, in a more general case, to Serscic's law [29] yields the model parameters 


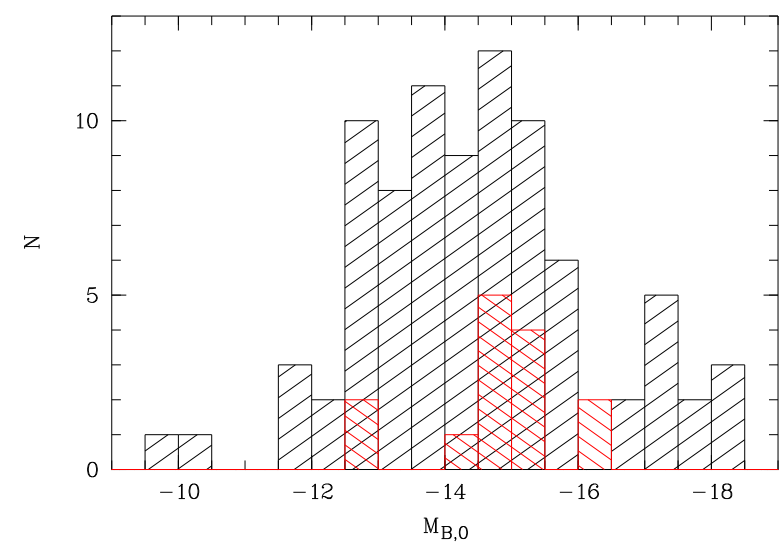

Figure 3. Distribution of the $B$-band absolute magnitudes for 85 galaxies of the Lynx-Cancer void. The red histogram shows the distribution for the subsample of the 14 so-called "inchoate" galaxies from [22].

that are also listed in Table 4: (2),(3) the central $g$ and $r$-band surface brightness; (4),(5) the central $B$-band surface brightness values (computed by Lupton's formulas [27]) uncorrected and corrected for Galactic extinction and inclination to the line of sight; (6) the scale length in arcsec; (7) the Sersic index.

\section{ANALYSIS AND DISCUSSION}

\subsection{Parameter Distributions}

We construct the observed distributions of the most important quantities for the sample studied to understand the full range of their variation. Figure 3 (the sparse hatching) shows the distribution of absolute magnitudes $M_{B, 0}$ for the void objects computed from our photometry and corrected for Galactic extinction. For comparison,

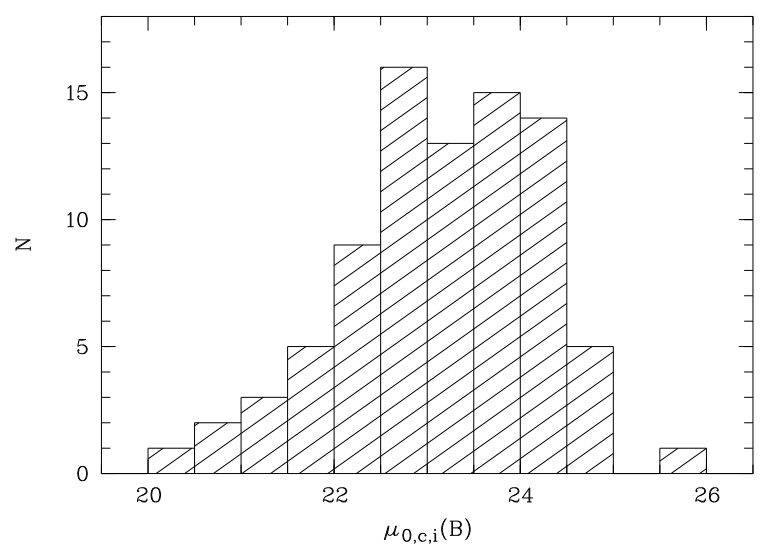

Figure 4. Distribution of the $B$-band central surface brightness corrected for Galactic extinction and inclination to the line of sight for 85 void galaxies.

we show the distribution of $M_{B, 0}$ for the subsample of the so-called "inchoate" galaxies from the ES survey [22] (red hatching in the same figure).

Garcia-Appadoo et al. [22] selected these 14 galaxies from the full list of 195 EquatorialSurvey objects on the basis of their peculiar properties - blue colors and high $\mathfrak{M}(\mathrm{HI}) / L_{g}$ ratios. The above authors overestimate the 21-cm line fluxes for some of these objects which leads to overestimated $\mathfrak{M}(\mathrm{HI}) / L_{g}$ ratios. We have corrected these values by taking into account the new published data, and the recomputed ratios are no longer so outstanding. In particular, the $\mathfrak{M}(\mathrm{HI}) / L_{B}$ ratio for the $\mathrm{NE}$ component of the H I 1225+01 (HIPEQ 1227+01) pair is now equal to 10.1 instead of the original estimate of 22 . In addition, to better understand the nature of the galaxies in this subsample, we searched their neighborhoods for the presence of massive galax- 


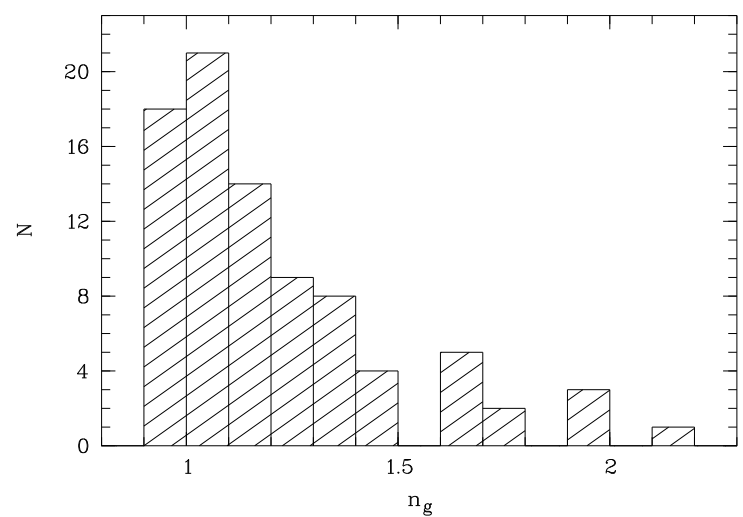

Figure 5. Distribution of the Sersic index [29] of the radial $g$-band surface brightness profile for 85 void galaxies.

ies. Of the 14 "inchoate" galaxies nine are separated by at least $1 \mathrm{Mpc}$ from the closest massive galaxy, and for the six most gas-rich objects the distances to the closest massive galaxies lie in the range of 2-5 Mpc. Thus, the galaxies of this subsample, like our void galaxies, show evident signs of isolation.

Figure 4 shows the distribution of the $B$-band central surface brightness $\mu_{0, c, i}(B)$ (corrected for Galactic extinction and inclination to the line of sight) for the studied subsample of the Lynx-Cancer void galaxies. The fraction of galaxies with $\mu_{0, c, i}(B)>23 \mathrm{~m}^{\mathrm{m}} 0 / \square^{\prime \prime}$ (LSB) in this subsample is equal to about $50 \%$. Figure 5 shows the distribution of the Sersic index $n_{g}$ determined by modeling the radial $g$-band brightness profile. The index $n_{g}$ is sufficiently close to 1 for almost two thirds of the objects, i.e., they have close-toexponential brightness profiles. The brightness profiles of the remaining galaxies with the

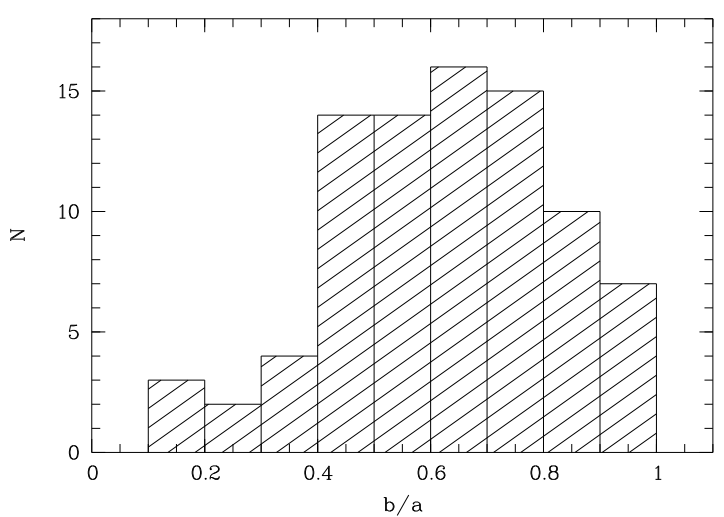

Figure 6. Distribution of the observed axial ratio $b / a$ for 85 void galaxies.

indices $n_{g}$ in the range of 1.2 to 2.1 flatten out appreciably towards the center. The distribution of the observed axial ratios $b / a$ in Fig. 6 shows that about $50 \%$ of the sample objects are tilted significantly to the line of sight $(b / a<0.65$ and, correspondingly, $i>50^{\circ}$ ). As we showed in our previous paper [15], because of the selectivity of the SDSS spectroscopic survey with respect to surface brightness many LSB galaxies in the void that are brighter than the formal SDSS cutoff threshold $r_{\text {petro }}<17.77$ [39] could lack velocity measurements. Because of the tilt to the line of sight, their apparent brightness is overestimated, making it more likely for them to be included in the spectroscopic part of the SDSS survey.

Figure 7 (the sparse hatching) shows the distribution of the hydrogen mass to luminosity ratio $\mathfrak{M}(\mathrm{HI}) / L_{B}$. The median value for 57 galaxies of our sample is $\mathfrak{M}(\mathrm{HI}) / L_{B} \sim 1.0$. Here, like in Fig. 3, we show the corresponding distribu- 


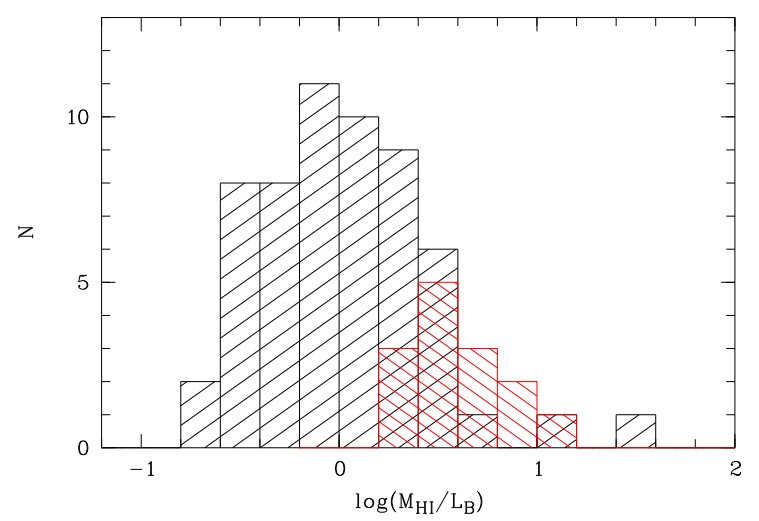

Figure 7. Distribution of the hydrogen mass to $B$-band luminosity ratio $\mathfrak{M}(\mathrm{HI}) / L_{B}$ (in solar units) for the void galaxies. The red histogram shows the distribution for the subsample of 14 "inchoate" galaxies from the total sample of the blind H I survey (ES) from [22].

tion for the "inchoate" galaxies from [22] (the red hatching). The median $\mathfrak{M}(\mathrm{HI}) / L_{B}$ ratio for this sample is equal to about 3.4. Garcia-Appadoo et al. [22] associate the term "inchoate" with the irregular shapes of these galaxies: the above authors assume that these systems are in the process of formation or at the beginning of their evolution. We show below that some of the galaxies in our sample also have peculiar properties and resemble "inchoate" objects from the ES sample (see Table 1). Furthermore, they are unusually blue $((B-V)<0.3)$ and the median value of their parameter $\mathfrak{M}(\mathrm{HI}) / L_{B} \sim 2.7$ is comparable with the corresponding parameter for the subsample of Garcia-Appadoo et al. [22].

Figure 8 shows the distribution of the gas mass fraction $f_{\text {gas }}$. The gas mass fraction for $59 \%$ of the void galaxies with available $\mathrm{H}$ I data lies in the range of $80-99 \%$. Such a high concentration

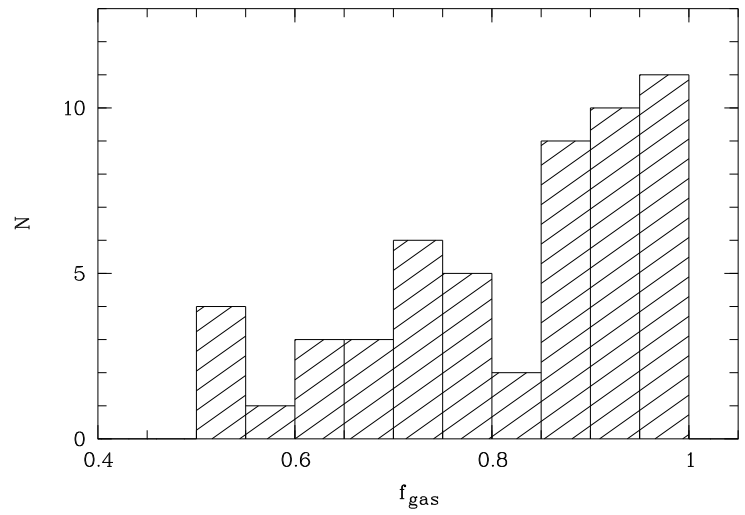

Figure 8. Preliminary distribution of the gas mass fraction for about half of the sample objects

(54 void galaxies) based on published H I data and observations made with the NRT radio telescope (see text for details).

of gas-rich objects in our sample indicates, as we already pointed out in our earlier paper [16], that the void population evolves more slowly than the galaxies in denser environments.

\subsection{Relations Between the Parameters of Void Galaxies}

In Fig. 9 we compare the absolute magnitude $M_{B, 0}$ and the central surface brightness $\mu_{0, c, i}(B)$ for the sample of 85 galaxies of the Lynx-Cancer void. Both parameters are corrected for Galactic extinction. The central surface brightness values are corrected for the galaxies' inclination to the line of sight. We estimate the central surface brightness values for galaxies with a substantial contribution from the central star-forming region and for galaxies with a "bulge" by extrapolating to the center of the underlying external disk 


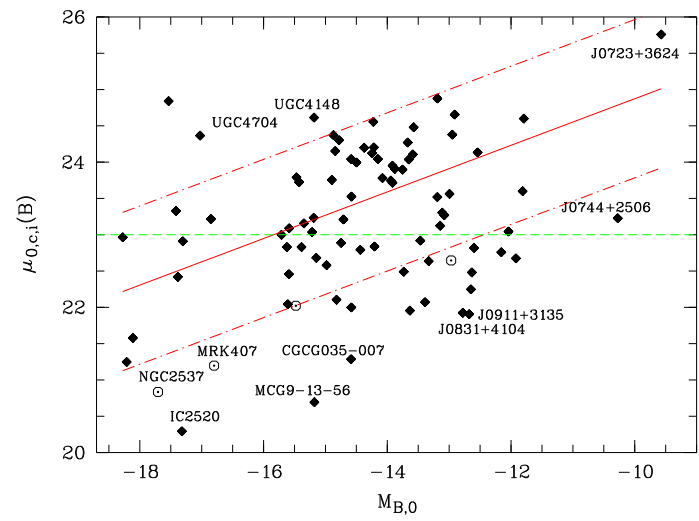

Figure 9. Relation between the absolute magnitude $M_{B, 0}$ and central surface brightness $\mu_{0, c, i}(B)$ for 85 galaxies of the Lynx-Cancer void. The horizontal green dashed line corresponds to the surface brightness level of $23^{\mathrm{m}} / \square^{\prime \prime}$, above which lies the domain of LSB galaxies. Linear regression (the red solid line) is performed based on the data points for 81 galaxies of the subsample considered. The red dash-dotted lines show the scatter of the subsample data points. Four BCGs (the open circles) were not used in the regression (see text).

profile. About half of the galaxies are classified as LSB, i.e., their corrected surface brightness is $\mu_{0, c, i}(B)>23^{\mathrm{m}} / \square^{\prime \prime}$ (lies above the green dashed line). Linear regression (the red solid line) is based on all the galaxies of the subsample except for four blue compact galaxies (BCG) with ongoing star formation. The standard deviation of individual galaxies from the general trend is rather large: $\sigma_{\mu_{0}}=0.97 / \square^{\prime \prime}$ (the red dashdotted lines). The open circles show the positions of the four BCGs. These rare low-mass galaxies have a higher central surface brightness and differ appreciably from the more typical late-type dwarf galaxies.

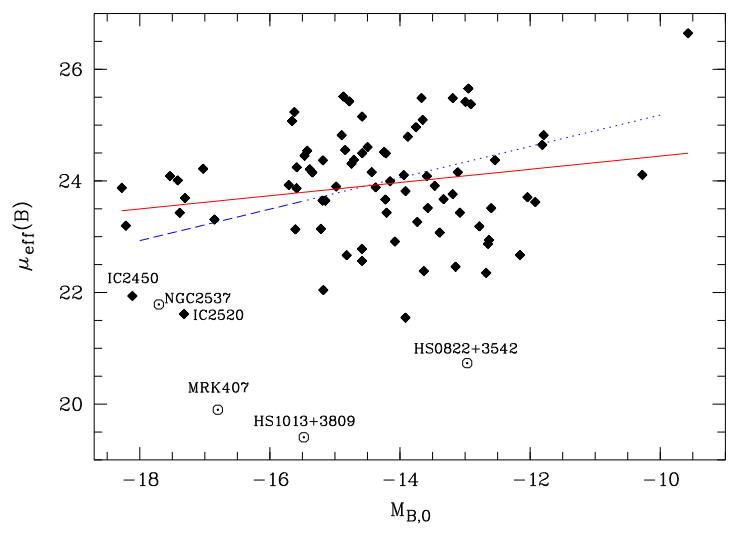

Figure 10. Relation between the absolute magnitude $M_{B, 0}$ and effective surface brightness $\mu_{\text {eff }}(B)$ for 85 galaxies of the Lynx-Cancer void. Linear regression (the red solid line) is based on all galaxies except for the four BCGs (the open circles). A similar line from Cross and Driver [13] (the blue dashed line) based on a sample of about 45000 galaxies and extrapolated into the domain of low luminosities down to $M_{B, 0}<-10$ (the blue dotted line) runs close to our relation in the common luminosity range $M_{B, 0}<-15.5$.

Figure 10 shows the relationship between the absolute magnitude $M_{B, 0}$ and the effective surface brightness $\mu_{\mathrm{eff}}(B)$. Both parameters are corrected for Galactic extinction. The red solid line shows the linear regression based on 81 void galaxies except for the four BCGs (its slope is equal to $k=0.12 \pm 0.06)$. The trend cannot be inferred reliably because of the large scatter of data points in the central part of the range. A similar line (shown by the blue dashes) based on the data for an extensive sample of galaxies (about 45000 objects) from Cross and Driver [13] and extrapolated into the domain of low luminosities (the blue dotted line) runs close to the center of the distribution for void galaxies but 
has a significantly higher slope $(k=0.28)$. This difference must be due to the higher dwarf fraction in the sample of void galaxies and greater diversity of their properties. Furthermore, the scatter around the regression line for the sample of Cross and Driver [13] is smaller than for void galaxies: $\sigma_{\mu} \sim 0{ }^{\mathrm{m}} 5 / \square^{\prime \prime}$ and $1^{\mathrm{m}} 0 / \square^{\prime \prime}$ respectively.

Figure 11 shows the relation between the effective surface brightness $\mu_{\mathrm{eff}}(g)$ and luminosity $\log L_{g}$ similar to the relation shown in the previous figure. Like in the previous figure, linear regression (the red solid line) is based on all galaxies except for the four BCGs. To compare our relations with similar relations based on the sample of 195 galaxies of the equatorial survey (ES) [22] selected by the 21-cm line emission recorded in the blind HIPASS H I survey, we show the linear regression for this sample in Fig. 11 by the blue dashed line. We extend this line to the domain of low luminosities, where we show it as a blue dotted line for display purposes. The open triangles indicate the 14 objects from the subsample of the so-called "inchoate" galaxies of Garcia-Appadoo et al. [22], which have blue colors and higher-than-usual hydrogen mass-to-luminosity ratios $((B-V) \leq 0.3$ and $\left.\mathfrak{M}(\mathrm{HI}) / L_{B}>1.8\right)$. The slope of our regression for void galaxies $(k=-0.35 \pm 0.16)$ is about 2.5 times lower than the corresponding slope for the ES-sample $(k=-0.83)$. Note that the sample of Garcia-Appadoo et al. [22] contains no objects with $\log L_{g}<7.5$. The slope of the regression based on the ES sample is rather close

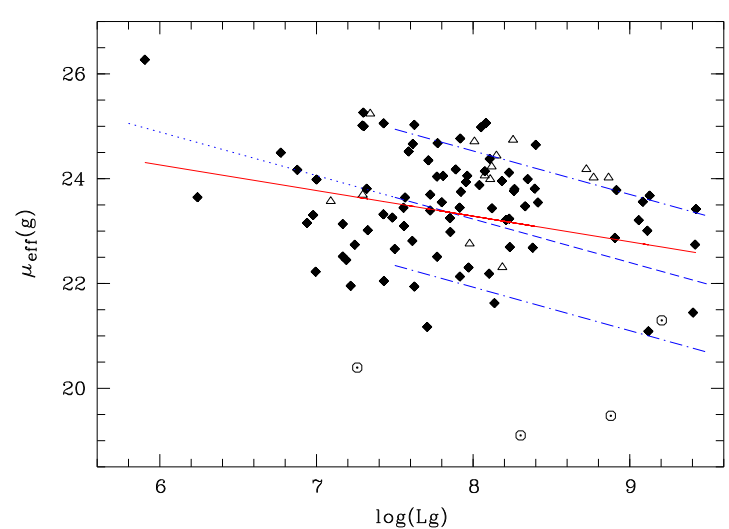

Figure 11. Relation between the effective surface brightness $\mu_{\text {eff }}(g)$ and $\log L_{g}$. The red solid line shows the linear regression based on the galaxies of our sample (the filled diamonds) except for the BCG galaxies (the open circles).

The blue dashed line shows the linear regression from Garcia-Appadoo et al. [22], the blue dotted line shows its extension to the domain of low luminosities, the blue dash-dotted lines show the scatter of the data points of the sample of Garcia-Appadoo et al. [22], and the open triangles show the group of 14 so-called "inchoate" galaxies pointed out by the above authors.

to the slope for the sample from [13]-namely, $k=-0.70$ if considering the correlation with the luminosity instead of the absolute magnitude. The scatter of the parameter $\mu_{\mathrm{eff}}(g)$ for void galaxies is, like the corresponding scatter for the sample of the ES survey, quite significant, reaching 3 or more magnitudes at a given luminosity.

The galaxies at the extremes of the range make the decrease of the surface brightness with decreasing luminosity in Figs. 10 and 11 more or less apparent. However, the rather large scatter of $\mu_{\mathrm{eff}}(g)$ for the void galaxies at the center 


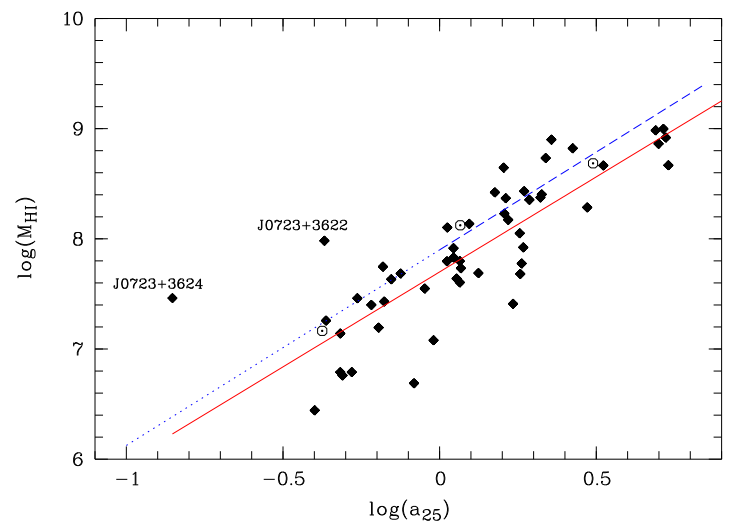

Figure 12. Relation between the optical radius $\log a_{25}(g)$ (kpc) and hydrogen mass in the galaxy $\log \mathfrak{M}(\mathrm{H}$ I) (in solar units). The red solid line shows the linear regression between these quantities (its slope is equal to 1.96) computed without the BCG galaxies shown by the open circles. For comparison, we show the relation from [22] (the blue dashed line) and its extension to the $a_{25}(g)<1 \mathrm{kpc}$ domain (the blue dotted line).

of the luminosity range indicates that voids are characterized not only by the general trend for the increase of the fraction of LSB galaxies but also by the presence of galaxies with a sufficiently high surface brightness which must be due to the enhanced star formation in the last several billion years. The large difference between the slopes of the regression for the galaxies of the Lynx-Cancer void and the ES sample may be due to the fact that the latter contains a substantial fraction of massive galaxies with enhanced light concentration at the center - a bulge or traces of a recent burst of star formation. This results in a shift of the average effective surface brightness at high luminosities in the ES sample and a larger re-

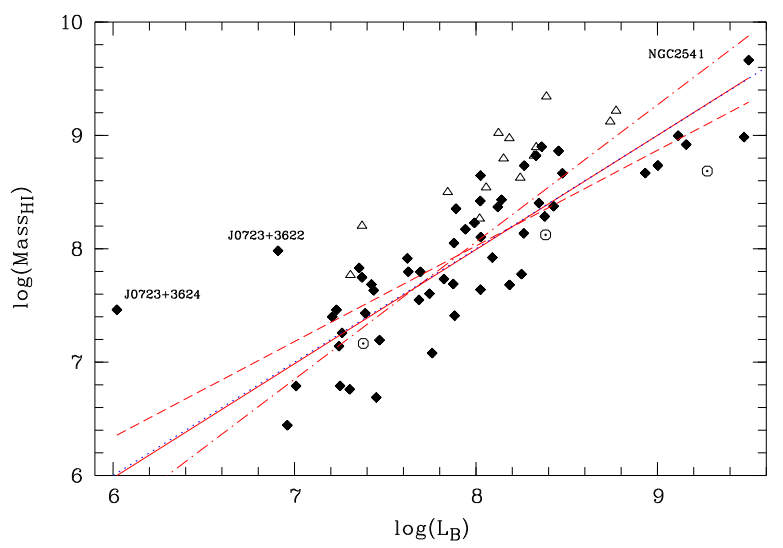

Figure 13. Relation between the hydrogen mass $\log \mathfrak{M}(\mathrm{HI})$ and luminosity $\log L_{B}$ in solar units. The red dashed line shows the linear regression throughout the entire luminosity range for the galaxies of our sample (the filled diamonds), the red solid line shows the linear regression computed without J0723+3622 and $\mathrm{J} 0723+3624$, and the red dash-dotted line shows the linear regression over the $\log L_{B}=7.0-8.6$ luminosity interval. All regressions were computed without BCG galaxies, which are shown by open circles. The open triangles show the group of the so-called "inchoate" galaxies from [22] which stand out because of their higher gas content. The blue dotted line corresponds to

$$
\mathfrak{M}(\mathrm{HI}) / L_{B}=1 .
$$

gression slope.

Figure 12 shows the relation between the optical radius $a_{25}(g)$ (kpc) and hydrogen mass of the galaxy $\mathfrak{M}(\mathrm{HI})$ (in solar units). In addition to the linear regression based on 85 galaxies of our sample (the red solid line, $k=1.96 \pm 0.16$ ), we also show a similar relation from [22] (the blue dashed line). They can be seen to agree well with each other over the common range of optical radii. Our data allow this relation to be extended to 
the domain of objects with $a_{25}(g)<1 \mathrm{kpc}$. The regression slope, which is close to 2 , reflects the simple physical fact that in the studied galaxies hydrogen is distributed throughout a rather flat disk and the characteristic radius of the H I disk closely correlates with that of the optical disk over a broad range of effective sizes. At the same time, the two objects (J0723+3624 and J0723+3622) with the highest hydrogen mass to $B$-band luminosity ratios $\mathfrak{M}(\mathrm{HI}) / L_{B}>10$ deviate strongly from the main trend.

Figure 13 shows the relation between the hydrogen mass $\mathfrak{M}(\mathrm{HI})$ and $B$-band luminosity $L_{B}$ (in solar units) for 54 void galaxies with known H I masses. The red dashed line shows the linear regression based on the galaxies of our sample (diamonds, $k 1=0.84 \pm 0.08$ ). The open triangles show the "inchoate" galaxies from [22] for comparison. The blue dotted line, where $\mathfrak{M}(\mathrm{HI}) / L_{B}=1$, separates the galaxies with a high gas fraction from the more typical galaxies with normal gas content. We use the same data to determine the dependence of the parameter $\mathfrak{M}(\mathrm{HI}) / L_{B}$ on $L_{B}$, whose regression slope is equal to $-0.16 \pm 0.08$. This slope is, within the rather large quoted errors, close to the slope of a similar dependence found by Pustilnik et al. [40] for BCGs in the Local Supercluster and in the voids.

A closer look at the figure discussed here suggests that the increase of $\mathfrak{M}(\mathrm{HI}) / L_{B}$ with decreasing $L_{B}$ is due mostly to the galaxies at the extremes of the range, including the two most gas-rich galaxies of the J0723+36 triplet. We also show other regression versions in the plot. The second regression is computed without the triplet members mentioned above: it is shown by the red solid line with the slope of $k 2=1.01 \pm 0.08$ (52 objects). The third regression is based on the galaxies within the narrow luminosity range of $\log L_{B}=7.0-8.6$ and shown by the red dash-dotted line with a slope of $k 3=1.21 \pm 0.12$ (45 objects). A comparison of the slope difference with its "combined error" indicates that the slopes differ at the level of $2.5-3 \sigma: \quad k 1-k 2=-0.17 \pm 0.056$, $k 1-k 3=-0.35 \pm 0.144$. We can thus conclude that the nature of the relation between the two global parameters of low-mass void galaxies over a wide luminosity range is so far not entirely clear. Extensive samples are needed, especially for the $\log L_{B}<7.5$ domain. Extending the analysis to other galaxies of this void and to low-mass galaxies from other nearby voids will increase the total number of galaxies involved by a factor of 2-3 and provide a better understanding of the discussed relation.

As is evident from Fig. 14, the $g$-band concentration index $C(g)=R_{90 \mathrm{~g}} / R_{50 \mathrm{~g}}\left(\right.$ where $R_{90}(g)$ and $R_{50}(g)$ are the radii containing $90 \%$ and half of the $g$-band flux of the galaxy) does not depend on luminosity $L_{g}$. The mean concentration index averaged over the galaxies of our sample is $C(g)=2.44$ (the red solid line) which is close to the value 2.32 for purely exponential disks. When computing our mean value, 


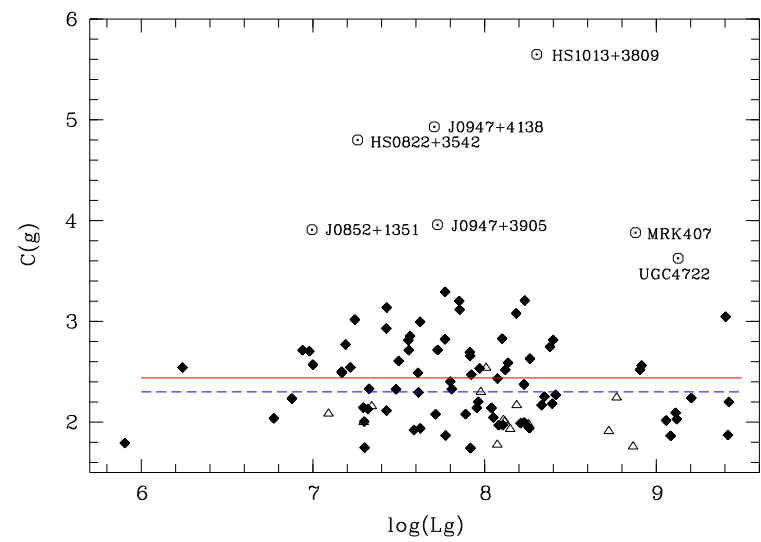

Figure 14. Relation between the luminosity $\log L_{g}$ and the $g$-band concentration index $C(g)$.

The red solid line shows the mean value $(R=2.44)$ averaged over all the objects of our sample (the filled diamonds). The blue dashed line shows the mean value $(R=2.3)$ from Garcia-Appadoo et al. [22] for their sample of 195 objects. The open triangles show the group of the so-called "inchoate" galaxies pointed out by the above authors.

we excluded, like in the above cases, the BCGs, very compact objects $(J 0947+4138$, J0947+3905, and J0852+1351), and the perturbed disk galaxy UGC 4722 with a tidal tale and an intense burst of star formation (all these objects are shown by open circles). We also show the mean concentration $C(g)=2.3$ for the galaxies from GarciaAppadoo et al. [22] (the blue dashed line) and the positions of their "inchoate" galaxies (the open triangles).

Figure 15 shows the relationship between the $g$-band luminosity $\operatorname{logarithm} \log L_{g}$ and the integrated color $(g-r)_{\text {tot }}$ for void galaxies. The red solid line shows the linear regression for this sample $(k=0.05 \pm 0.02)$. For comparison, we show

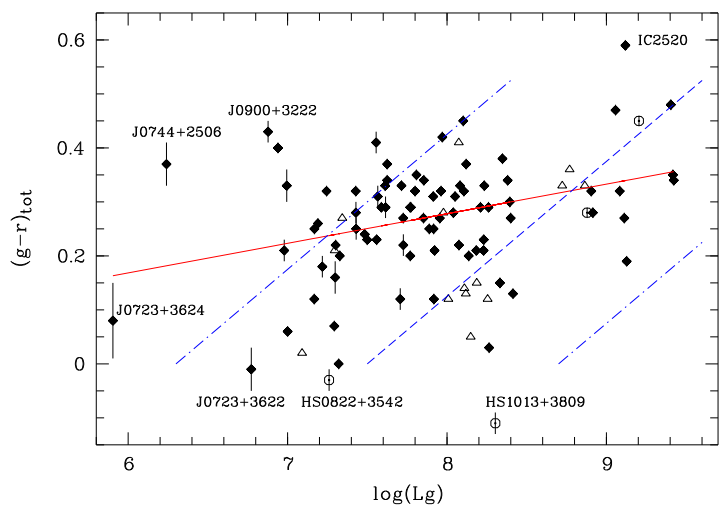

Figure 15. Relation between the $g$-band luminosity $\log L_{g}$ and integrated color $(g-r)_{\text {tot }}$. The red solid line shows the linear regression based on all the objects of our sample (the filled diamonds) except for the BGCs (the open circles). The blue dashed line shows the linear regression from Garcia-Appadoo et al. [22], the blue dash-dotted lines show the scatter of the data points of their sample, and the open triangles show the group of "inchoate" galaxies pointed out by the above authors.

by the blue dashed line the linear regression for the galaxies of the ES survey $(k=0.25)$ and the positions of the "inchoate" galaxies from [22] (the open triangles). Whereas the galaxies of the ES sample exhibit a strong trend, the $(g-r)_{\text {tot }}$ colors (like the parameter $\mu_{\mathrm{eff}}(g)$ in Fig. 11) on the average vary only slightly with decreasing luminosity: from 0.35 to about 0.15 , albeit with a large scatter. To understand the nature of this scatter better, galaxies with extreme $(g-r)_{\text {tot }}$ parameters should be studied in more detail.

Figure 16 shows the relationship between the stellar mass fraction $\left(\log \left(1-f_{\text {gas }}\right)\right)$ and integrated color $(g-r)_{\text {tot }}$. The red solid line shows the linear regression between these parameters 


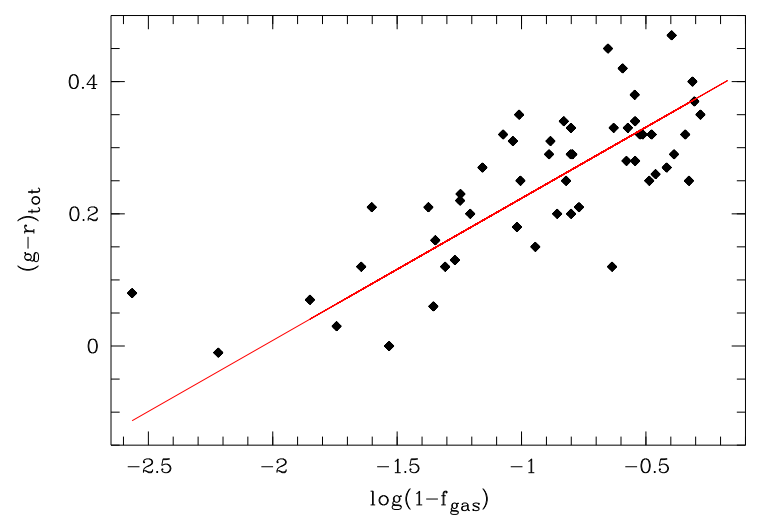

Figure 16. Relation between the stellar mass fraction $\left(\log \left(1-f_{\text {gas }}\right)\right)$ and integrated color $(g-r)_{\text {tot. }}$ The red solid line shows the linear regression based on the objects of our sample.

$(k=0.215 \pm 0.024)$. As expected, in this case the relation shows up more conspicuously than in the previous figure, because the coefficient $\Upsilon$ used to estimate the stellar mass from luminosity depends on the $(g-i)$ color (which correlates with $g-r)$-see Section 3.2. The bluer the color, the smaller the stellar mass, and, for a fixed gas mass, the smaller the stellar mass fraction.

Figure 17 shows the relationship between the stellar mass fraction $\left(\log \left(1-f_{\text {gas }}\right)\right)$ and the oxygen abundance in the interstellar medium (similar to metallicity), $12+\log (\mathrm{O} / \mathrm{H})$, for the void galaxies for which this parameter has been measured. Like in the previous figures, the red solid line shows the linear regression $(k=0.32 \pm 0.10)$. The apparent trend of the decrease of metallicity with decreasing stellar mass fraction is consistent with what is to be expected in the so-called "closed box" model of galaxy evolution (i.e., evolution without exchange with the surrounding

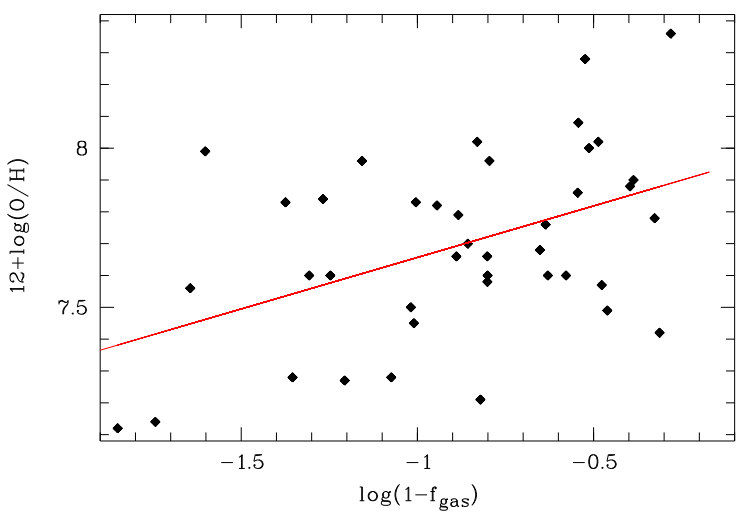

Figure 17. Relation between the stellar mass fraction $\left(\log \left(1-f_{\text {gas }}\right)\right)$ and metallicity $12+\log (\mathrm{O} / \mathrm{H})$. The red solid line shows the linear regression based on the objects of our sample.

medium). However, the scatter of the parameter $\mathrm{O} / \mathrm{H}$ is so large that the "closed box" approximation is often invalid in void galaxies, including LSBD galaxies.

\subsection{Two-Color Diagrams and Age Estimates}

In Figs. 1 and 2 we compare the colors of the outer regions of 81 void galaxies with PEGASE2 models for two extreme cases of the star-formation (SF) law: instantaneous (inst) SF and continuous (const) SF with a constant rate. We adopt two initial stellar mass functions (IMF): the standard Salpeter IMF and the IMF of Kroupa. For the sake of illustration we show the tracks for the metallicity of $Z=0.002$, which lies approximately in the middle of the range of known metallicities of void galaxies. The contribution of nebular emission may be significant for the four BCGs in our sample. We excluded these objects from further analysis because the 
Table 5. Parameters of peculiar galaxies in the void

\begin{tabular}{l|c|c|c|c|c|c|c|c}
\hline Parameter & $\mathrm{J} 0723+3622$ & $\mathrm{~J} 0723+3624$ & $\mathrm{~J} 0737+47$ & $\mathrm{~J} 0812+48$ & $\mathrm{~J} 0926+33$ & $\mathrm{~J} 0929+25$ & $\mathrm{~S} 0822+3545$ & $\mathrm{U} 3966$ \\
\hline$T_{\text {old }}, \mathrm{Gyr}$ & $1-2$ & $2-2.5$ & $2-3$ & $4-6$ & $3-3.5$ & $3-5$ & $1-3$ & $2.5-3$ \\
$f_{\text {gas }}$ & 0.997 & 0.997 & 0.96 & 0.94 & 0.99 & 0.95 & 0.97 & 0.98 \\
$12+\log (\mathrm{O} / \mathrm{H})$ & - & - & 7.28 & 7.27 & 7.12 & $7.2:$ & - & 7.56 \\
$M_{B}$ & -11.79 & -9.57 & -12.54 & -13.08 & -12.91 & -12.95 & -13.11 & -14.58 \\
\hline
\end{tabular}

estimates of their stellar population colors are uncertain.

The colors of the outer regions of 77 of the 81 void galaxies studied here agree rather well with the PEGASE2 evolutionary tracks for the case of continuous star formation. The time $T_{\mathrm{SF}}$ elapsed since the onset of the star-formation (or in other terms, the age of a galaxy) spans a wide range, approximately from 1 to 14 Gyr. Most of these galaxies better fit the tracks computed with the Kroupa IMF. The colors of the outer parts of the majority of the objects correspond to typical galactic ages: $T \sim 10-13$ Gyr. However, the colors of the outer regions of seven objects correspond to the times $T_{\mathrm{SF}}$ as small as about 1-3.5 Gyr. The colors of six other galaxies correspond to intermediate times $T_{\mathrm{SF}} \sim 4-6.5 \mathrm{Gyr}$. We discuss these galaxies and their peculiarities in more detail in the next section.

Only for four galaxiesJ0744+2506, IC 2450, and CGCG035-007-their rather red $(g-r \sim 0.5-0.6)$ colors, unlike those of the remaining 77 galaxies, can be interpreted as a result of a rather short ("instantaneous") and very old (about 10 Gyr) star formation episode, typical of elliptical galaxies. However, this void [17, 18, 41-44]. their morphology is inconsistent with such a hypothesis. The presence of emission regions either near the center, like in J0744+2506 and IC 2450, or shifted towards the edge, like in J0928+284 and CGCG 035-007, is indicative of recent star formation caused either by external perturbation or by the infall of fresh gas. These galaxies deserve a more thorough investigation as objects whose outer regions have colors atypical of the sample studied. In particular, it is important to understand what fraction of the fainter of such objects remains undiscovered because of observational selection if the star formation in them has not been triggered by any external factors over the past several tens of Myr.

\subsection{Peculiar Galaxies of the Void}

The peculiar properties of some galaxies with non-cosmological times elapsed since the onset of the main star formation episode $(\mathrm{SAO} 0822+3542, \quad \mathrm{UGC} 5340=\mathrm{DDO} 68$ $\mathrm{J} 0926+3343, \quad \mathrm{~J} 0723+3622, \quad \mathrm{~J} 0723+3624, \quad$ and $\mathrm{J} 0737+4724)$ were already pointed out in the papers dedicated to the individual galaxies of$$
\text { this vid }[17,18,41-41 \text {. }
$$ 
Here we give for these galaxies an independent corroboration of the blue colors of their peripheral regions which corresponds to short times elapsed since the onset of continuous star formation: $T_{\mathrm{SF}} \lesssim 3.5$ Gyr. For two other galaxiesUGC 3966, UGC 4117-such data have been obtained for the first time. The colors of the outer regions of UGC 3672, UGC 3860, J0812+4836, J0929+2502, UGC 5272 and its companion UGC 5272b also correspond to non-cosmological times in the $T_{\mathrm{SF}}$ rangeof $\sim 3-6.5 \mathrm{Gyr}$.

Table 5 summarizes the evolutionary parameters for eight galaxies which can be classified as unevolved based on the combination of their properties. Their gas mass fractions are equal to 94-99.7\%, and their metallicities $12+\log (\mathrm{O} / \mathrm{H})$ are 2-5 times lower than those of typical irregular dwarf galaxies of the same luminosity but residing in environments with intermediate or high density of galaxies (see [41-45]), whereas the colors of the visible old stellar population in these objects correspond to the epoch of the onset of star formation $T_{\mathrm{SF}} \lesssim 1-5$ Gyr. We place the object J0723+3624 with $M_{B}=-9.57$ in the same group of galaxies. The UGC 4117 galaxy has blue peripheral colors corresponding to $T_{\mathrm{SF}} \sim 2$ Gyr but does not fit into the list of peculiar galaxies of the void because its metallicity $12+\log (\mathrm{O} / \mathrm{H})=7.82$ does not differ very much from that expected for its luminosity $\left(M_{B}=-15.6\right)$. The same is true for the galaxies UGC 3672, UGC 3860, UGC 5272, and UGC 5272b with peripheral ages ranging from
5 to 6 Gyr. New Hubble Space Telescope data for UGC 5340 (DDO 68) [46] confirm the suggestions of Pustilnik et al. [42] and Ekta et al. [47] that this galaxy is made up of two merging components with very different properties and that this fact should be taken into account when associating the object with the group of peculiar galaxies. The more massive central component with $M_{B} \sim-16$ has an old stellar population with the metallicity five times lower than solar and hence is a rather typical late-type galaxy. The stars in the much less massive component (UGC 5340b), extending along the eastern edge of the more massive component, have metallicities no greater than $Z_{\odot} / 20$ which is consistent with the gas metallicity $12+\log (\mathrm{O} / \mathrm{H})=7.14$ estimated for the H II regions of the galaxy. The ages of most or all of its stars do not exceed 2 Gyr. However, thus far the mass or luminosity of the less massive component cannot be estimated.

A closer analysis of the properties of the most peculiar galaxies mentioned above suggests that their fraction is much higher among less luminous galaxies. However, this may be an accidental result due to the statistically small number of bright void galaxies. The hypothesis about a relation between the luminosity and the fraction of unevolved galaxies in the void can be tested using the statistical criterion " $2 \times 2$ contingency table" [48]), which is widely used in biology and applied studies. A detailed description of this criterion can be found in the paper by Pustilnik 
et al. [49], who used it for astronomical applications.

The idea of the method consists of analyzing the numbers in a $2 \times 2$ contingency table, which correspond to different combinations of two properties of the elements of the sample. In our case we adopt the property $Y$ of being a low-luminosity galaxy with $M_{B}>M_{\text {faint }}$, where $M_{\text {faint }}$ is the threshold value based on certain considerations. Thus, the property $Y$ breaks the sample into the $Y$ and non- $Y$ subsamples. Similarly, the second property $Z$ is that of being an unevolved galaxy according to the above criteria. Correspondingly, non- $Z$ means that the object is a galaxy with a more standard visible stellar population. To test the zero hypothesis $h_{0}$ that the two properties in the sample considered are independent of each other, we must compose a $2 \times 2$ table in the following form:

$\begin{array}{lccc}\text { Property } & Y & \text { non- } Y & \text { Total } \\ Z & m & n-m & n \\ \text { non- } Z & M-m & N-n-(M-m) & N-n \\ \text { Total } & M & N-M & N\end{array}$

Here $m, n-m, M-m$, and $N-n-(M-m)$ are the numbers of galaxies in the sample having the property combinations $(Y, Z)$, (non- $Y, Z)$, $(Y$, non- $Z)$, and (non- $Y$, non- $Z$ ) respectively. As is shown in the book [48] (pp. 77-78), if the properties $Y$ and $Z$ are independent of each other, the probability of obtaining the contingency table with such numbers is described by a hypergeometric distribution, which at $N>25$ can be well approximated by the so-called incom- plete beta function $I_{x}(a, b)$, where the parameters $x, a$, and $b$ can be expressed in terms of the numbers in the $2 \times 2$ contingency table by the formulas (27)-(30) on p. 74 of the book [48] (see also Appendix to [49]).

If there is no real correlation in the sample of void galaxies between the properties of low luminosity and "small" age, then in the case of the observed distribution of absolute magnitudes and whatever threshold $M_{\text {faint }}$ is adopted, the numbers in the contingency table should correspond to a relatively low probability of rejecting the null hypothesis.

To account more fully for all the available information on the unevolved galaxies of the Lynx-Cancer void sample, we considered the object J0723+3624 with the absolute magnitude $M_{B}=-9.57$ to be an unevolved object when analyzing the $2 \times 2$ contingency table. Despite the failure to measure the peripheral colors of this very small galaxy, which is a member of the unusual triplet in the central part of the void, the record high gas mass fraction of this object (0.997) and its blue integrated color result in age estimates of less than 2-3 Gyr in any reasonable evolutionary scenario (Fig. 7 in [18]). In view of the above, the $2 \times 2$ contingency table for $M_{\text {faint }}=-13.15$ is:

$\begin{array}{cccc}\text { Property } & Y & \text { non- } Y & \text { Total } \\ Z & 6 & 12 & 18 \\ \text { non- } Z & 2 & 65 & 67 \\ \text { Total } & 8 & 77 & 85\end{array}$

The probability of the table with these values 
computed by the appropriate formulas for the incomplete beta function yields a statistically significant result: the probability of rejecting the null hypothesis is $P=0.9993$. This result should be interpreted as the existence of a significant statistical relation between the two properties in the sense that the fraction of unevolved galaxies is significantly higher in the group with the absolute magnitudes fainter than $M_{\text {faint }}=-13.15$ (a simple comparison of the fractions 6/18 and $2 / 67$ indicates a difference of more than one order of magnitude). For this computation we included the unevolved component UGC 5340b in the sample, assuming that its $M_{B}$ is brighter than -13.15 . Otherwise the significance level would be even higher.

The discovery of such a relation has two important implications. The first one is associated with the understanding of the physics of the processes resulting in unevolved galaxies appearing only among sufficiently low-mass objects. The second implication is a methodological one. The correlation found indicates that to search effectively for such objects in the voids, one must study galaxies with absolute magnitudes fainter than or near the "threshold."

Let us return to the situation with the blue colors of the peripheral regions of some galaxies, where the parameter $\mathrm{O} / \mathrm{H}$ is only slightly lower than expected for their luminosity (UGC 3672, $3860,4117,5272$, and 5272b). These objects do not resemble unevolved galaxies, but their unusual colors require further discussion. They can be explained by a relatively recent perturbation (on a time scale of 2-6 Gyr) which resulted in an increased rate of star formation, the traces of which are now also visible in their outer regions. The optical morphology of the UGC 3672, UGC 3860, and UGC 4117 galaxies and that of the pair UGC 5272/5272b is indeed indicative of a significant perturbation and/or ongoing interaction. Further HI mapping of these objects should provide more compelling evidence for their perturbed state.

\section{CONCLUSIONS}

To summarize our study of void galaxies including their statistical analysis and a comparison with other data and samples, we formulate the following results and conclusions.

(1) We determined the photometric parameters in the $u, g, r, i$ bands (integrated magnitudes and colors, effective radii and the corresponding surface brightness values, the optical and Holmberg radii) for 85 galaxies of the Lynx-Cancer void based on their images adopted from the SDSS database. We analyzed the radial surface brightness profiles to determine the central surface brightness levels and scale lengths of the model disks.

(2) We compared the $(u-g),(g-r),(r-i)$ color indices of the outer regions of the galaxies outside the regions of recent star formation with the PEGASE2 evolutionary tracks and es- 
timated the time $T_{\mathrm{SF}}$ elapsed since the onset of the star-formation. For about $85 \%$ of the galaxies, these time scales lie in the range of $T \sim 10-13$ Gyr, which is typical of galaxies in denser environments. The colors of 13 galaxies imply much smaller $T_{\mathrm{SF}}$. The corresponding times for seven galaxies lie in the range of 13.5 Gyr, and those of the six remaining galaxies range approximately from 4 to $6.5 \mathrm{Gyr}$.

(3) Most of the galaxies with unusual colors of outer regions have very low gas metallicities $(12+\log (\mathrm{O} / \mathrm{H}) \lesssim 7.4$ which are $2-5$ times lower than expected for their luminosity) and the highest values of the empirical gas content parameter $\mathfrak{M}(\mathrm{HI}) / L_{B} \gtrsim 2.5$. We estimated the stellar masses of the galaxies of this group and found their gas mass fractions to be in the range of $94-99 \%$ which is the highest value of this parameter ever determined. By their properties these galaxies are unevolved.

(4) A comparison of the statistical relations between the observed properties of the galaxies in the Lynx-Cancer void and galaxies from the Equatorial Survey (ES) initially selected by their HI line emission shows the similarity of the two samples throughout the common luminosity range. However, void galaxies contain a significant fraction of objects that do not follow the common trends and relations. A small group of the so-called "inchoate" galaxies in the ES survey exhibits unusual properties. More detailed studies will probably show what part of them are analogs of unusual galaxies in the voids.

(5) The group of unevolved galaxies accounts for about $15 \%$ of all the low surface brightness dwarf galaxies in the void and is represented substantially more often among low luminosity galaxies $\left(M_{B}>-13.2\right)$, where their fraction amounts to about $30 \%$. This fact confirms that the environment has a stronger effect on less massive galaxies and provides a clue to an efficient search for such unusual galaxies.

\section{ACKNOWLEDGMENTS}

We are grateful to D. I. Makarov for his numerous useful critical comments and suggestions, which helped improve the paper substantially. Yu. A. Perepelitsyna and S. A. Pustilnik acknowledge the support of the Russian Foundation for Basic Research (grants No. 11-02-00261, 13-02-90407, and 13-02-90734) and partial support of the Federal target programme Research and Pedagogical Cadre for Innovative Russia (proposal No. 2012-1.5-12-000-1011-004, agreement number 8523). A. Yu. Kniazev acknowledges the support of the National Research Foundation (NRF) of South Africa. We are grateful to the SDSS consortium for the spectroscopic, photometric, and auxiliary information on the studied galaxies available from the SDSS database [20]. 
1. M. Jöeveer, J. Einasto, and E. Tago, Monthly Notices Roy. Astronom. Soc. 185, 357 (1978).

2. R. P. Kirshner, A. Oemler Jr., P. L. Schechter, and S. A. Shectman, Astrophys. J. 248, L57 (1981).

3. A. D. Montero-Dorta and F. Prada, Monthly Notices Roy. Astronom. Soc. 399, 1106 (2009).

4. V. de Lapparent, in Proc. Les Houches Summer School, Session LX, Cosmology and Large Scale Structure, Ed. by R. Schaeffer, J. Silk, M. Spiro, and J. Zinn-Justin (Elsevier Sci. Publ. Co., Amsterdam, 1996), p. 107.

5. P. J. E. Peebles, Astrophys. J. 557, 459 (2001).

6. A. V. Tikhonov and A. A. Klypin, Monthly Notices Roy. Astronom. Soc. 395, 1915 (2009).

7. R. R. Rojas, M. S. Vogeley, F. Hoyle, and J. Brinkmann, Astrophys. J. 617, 50 (2004).

8. R. R. Rojas, M. S. Vogeley, F. Hoyle, and J. Brinkmann, Astrophys. J. 624, 571 (2005).

9. S. G. Patiri, F. Prada, J. Holtzman, et al., Monthly Notices Roy. Astronom. Soc. 372, 1710 (2006).

10. F. Hoyle, M. S. Vogeley, and D. Pan, Monthly Notices Roy. Astronom. Soc. 426, 3041 (2012).

11. K. Kreckel, M. R. Joung, and R. Cen, Astrophys. J. $\mathbf{7 3 5}, 132$ (2011).

12. J. E. Einasto, Colloquim on Cosmic Voids, http: //www . astro.rug.nl/ weygaert/ /knawvoid.program.php

13. N. Cross and S. P. Driver, Monthly Notices Roy. Astronom. Soc. 329, 579 (2002).

14. J. C. Mihos, S. S. McGaugh, and W. J. G. de Block, Astrophys. J. 477, L79 (1997).

15. S. A. Pustilnik and A. L. Tepliakova, Monthly Notices Roy. Astronom. Soc. 415, 1188 (2011).
16. S. A. Pustilnik, A. L. Tepliakova, and A. Y. Kniazev, Astrophysical Bulletin 66, 255 (2011).

17. S. A. Pustilnik, J.-M. Martin, A. L. Tepliakova, and A. Y. Kniazev, Monthly Notices Roy. Astronom. Soc. 417, 1335 (2011).

18. J. N. Chengalur and S. A. Pustilnik, Monthly Notices Roy. Astronom. Soc. 428, 1579 (2013).

19. K. Kreckel, E. Platen, M. A. Aragon-Calvo, et al., Astronom. J. 144, 16 (2012).

20. K. N. Abazajian, J. K. Adelman-McCarthy, M. A.Agüeros, et al., Astrophys. J. Suppl. 182, 543 (2009).

21. M. Fioc, B. Rocca-Volmerange, arXiv:astro-ph/9912179 (1999).

22. D. A. Garcia-Appadoo, A. A. West, J. J. Dalcanton, et al., Monthly Notices Roy. Astronom. Soc. 394, 340 (2009).

23. M. J. Meyer, M. A. Zwaan, R. L. Webster, et al., Monthly Notices Roy. Astronom. Soc. 350, 1195 (2004).

24. M. P. Haynes, R. Giovanelli, A. M. Martin, et al., Astrophys. J. 142, 170 (2011).

25. D. G. York, J. Adelman, J. E. Anderson, et al., Astronom. J. 120, 1579 (2000).

26. A. Y. Kniazev, E. K. Grebel, S. A. Pustilnik, et al., Astronom. J. 127, 704 (2004).

27. R. Lupton, http://www.sdss.org/dr7/ /algorithms/sdssUBVRITransform.html\# \#Lupton2005

28. E. Holmberg, Lund Medd. Astron. Obs. Ser. 136, 1 (1958).

29. J. L. Sersic, Boletin de la Asociacion Argentina de Astronomia 6, 99 (1963).

30. E. F. Schlafly and D. P. Finkbeiner, Astrophys. J. 737, 103 (2011). 
31. L. D. Matthews, J. S. Gallagher, W. van Driel, Astronom. J. 118, 2751 (1999).

32. P. C. van der Kruit and L. Searle, Astronom. and Astrophys. 95, 105 (1981).

33. S. Roychowdhury, J. N. Chengalur, I. D. Karachentsev, and E. I. Kaisina, Monthly Notices Roy. Astronom. Soc. 436, 104 (2013).

34. E. E. Salpeter, Astrophys. J. 121, 161 (1955).

35. P. Kroupa, C. A. Tout, and G. Gilmore, Monthly Notices Roy. Astronom. Soc. 262, 545 (1993).

36. D. L. Tucker, S. Kent, M. W. Richmond, et al., Astronomische Nachrichten 327, 821 (2006).

37. M. S. Roberts, Astronom. J. 74, 859 (1969).

38. S. Zibetti, S. Charlot, and H.-W. Rix, Monthly Notices Roy. Astronom. Soc. 400, 1181 (2009).

39. V. Petrosian, Astrophys. J. 209, 1 (1976).

40. S. A. Pustilnik, J.-M. Martin, W. K. Huchtmeier, et al., Astronom. and Astrophys. 389, 405 (2002).

41. S. A. Pustilnik, A. Y. Kniazev, A. G. Pramsky, et al., Astronom. and Astrophys. 409, 917 (2003).
42. S. A. Pustilnik, A. Y. Kniazev, and A. G. Pramsky, Astronom. and Astrophys. 443, 91 (2005).

43. S. A. Pustilnik, A. L. Tepliakova, and A. Y. Kniazev, Astronomy Letters 34, 457 (2008).

44. S. A. Pustilnik, A. L. Tepliakova, A. Y. Kniazev, and A. N. Burenkov, Monthly Notices Roy. Astronom. Soc. 401, 333 (2010).

45. S. A. Pustilnik, A. G. Pramskij, and A. Y. Kniazev, Astronom. and Astrophys. 425, 51 (2004).

46. N. A. Tikhonov, O. A. Galazutdinova, and V. S. Lebedev, Astronomy Letters 40, 1 (2014).

47. Ekta, J. N. Chengalur, and S. A. Pustilnik, Monthly Notices Roy. Astronom. Soc. 391, 881 (2008).

48. L. N. Bolshev and N. V. Smirnov, Tables of Mathematical Statistics (Nauka, Moscow, 1983) [in Russian].

49. S. A. Pustilnik, A. V. Ugryumov, V. A. Lipovetsky, et al., Astrophys. J. 443, 499 (1995). 
Table 1. Basic parameters of the galaxies in the Lynx?Cancer void.

\begin{tabular}{|c|c|c|c|c|c|c|c|c|c|c|c|c|c|c|c|}
\hline & $\begin{array}{l}\text { Name or } \\
\text { prefix } \\
(1)\end{array}$ & $\mid \begin{array}{c}\text { Name in brief } \\
\text { IAU format } \\
(2)\end{array}$ & $\begin{array}{c}\text { Type } \\
(3)\end{array}$ & $\mid \begin{array}{c}V_{\text {hel }} \\
\mathrm{km} / \mathrm{s} \\
(4)\end{array}$ & $\begin{array}{c}\text { Dist } \\
\quad \mathrm{Mpc} \\
(5)\end{array}$ & $\begin{array}{c}A_{\mathrm{B}}^{\mathrm{NED}} \\
\operatorname{mag} \\
(6)\end{array}$ & $\left|\begin{array}{c}B_{\text {tot }} \\
\text { mag } \\
(7)\end{array}\right|$ & $\mid \begin{array}{c}M_{\mathrm{B}, 0} \\
\operatorname{mag} \\
(8)\end{array}$ & $\begin{array}{c}B_{\text {tot }}^{\mathrm{NED}} \\
\text { mag } \\
(9)\end{array}$ & $\left|\begin{array}{c}\mathfrak{M}(\mathrm{HI}) \\
10^{7} M_{\odot} \\
(10)\end{array}\right|$ & $\begin{array}{c}\mathfrak{M}(\mathrm{HI}) / L_{\mathrm{B}} \\
(11)\end{array}$ & $\mid \begin{array}{c}\mathfrak{M}_{\text {star }} \\
10^{7} M_{\odot} \\
(12)\end{array}$ & $\left|\begin{array}{l}f_{\text {gas }} \\
(13)\end{array}\right|$ & $\begin{array}{c}12+\log (\mathrm{O} / \mathrm{H}) \\
(14)\end{array}$ & $\mid \begin{array}{l}\mathrm{b} / \mathrm{a} \\
(15)\end{array}$ \\
\hline 1 & HIPASSJ0626+24 & J0626+2439 & Scd & $|1473|$ & 23.04 & 1.79 & 17.98 & $\mid-15.62$ & 17.60 & - & - & 5.53 & - & - & 0.59 \\
\hline 2 & UGC3600 & J0655+3905 & $\operatorname{Im}$ & 412 & 9.30 & 0.29 & 15.92 & -14.22 & 16.18 & 11.23 & 1.48 & 1.91 & 0.89 & 7.66 & 0.27 \\
\hline 3 & UGC3672 & $\mathrm{J} 0706+3019$ & $\mathrm{Im}$ & 994 & 16.93 & 0.32 & 16.04 & -15.43 & 15.40 & 79.50 & 3.45 & 2.60 & 0.98 & 7.99 & 0.55 \\
\hline 4 & SDSS & $\mathrm{J} 0713+2926$ & $\operatorname{Im}$ & 950 & 16.26 & 0.31 & 16.66 & -14.71 & 16.79 & - & - & 2.66 & - & - & 0.63 \\
\hline 5 & SDSS & $\mathrm{J} 0723+3621$ & $\mathrm{Sm}$ & 917 & 16.00 & 0.23 & 17.01 & -14.25 & 17.01 & 22.60 & 2.91 & 2.76 & 0.92 & 7.45 & 0.31 \\
\hline 6 & SDSS & $\mathrm{J} 0723+3622$ & dI & 970 & 16.00 & 0.23 & 19.46 & -11.79 & 19.31 & 9.61 & 11.84 & 0.07 & 0.99 & - & 0.71 \\
\hline 7 & SDSS & $\mathrm{J} 0723+3624$ & dI & 938 & 16.00 & 0.23 & 21.68 & \begin{tabular}{|l}
-9.57 \\
\end{tabular} & 21.56 & 2.90 & 27.62 & 0.01 & 0.99 & - & 0.47 \\
\hline 8 & UGC3860 & $\mathrm{J} 0728+4046$ & $\operatorname{Im}$ & 354 & 7.81 & 0.25 & 15.21 & -14.50 & 14.96 & 16.92 & 1.73 & 2.13 & 0.91 & 7.83 & 0.56 \\
\hline 9 & UGC3876 & $\mathrm{J} 0729+2754$ & SAd & 854 & 15.01 & 0.19 & 13.77 & -17.31 & 13.70 & 99.48 & 0.77 & 78.17 & 0.63 & 7.88 & 0.58 \\
\hline 10 & SDSS & $\mathrm{J} 0730+4109$ & dI & 874 & 15.70 & 0.27 & 16.67 & -14.58 & 16.67 & 4.36 & 0.41 & 2.36 & 0.71 & 8.02 & 0.59 \\
\hline 11 & SDSS & $\mathrm{J} 0737+4724$ & LSB & 476 & 10.42 & 0.47 & 18.02 & -12.54 & 18.06 & 2.51 & 1.56 & 0.11 & 0.97 & 7.28 & 0.47 \\
\hline 12 & UGC3966 & $\mathrm{J} 0741+4006$ & $\operatorname{Im}$ & 361 & 8.64 & 0.22 & 15.32 & -14.58 & 14.44 & 44.26 & 4.18 & 1.22 & 0.98 & 7.56 & 0.86 \\
\hline 13 & DDO47 & $\mathrm{J} 0741+1648$ & IBsm & 272 & 8.04 & 0.14 & 14.89 & -14.78 & 13.62 & - & - & 3.59 & - & - & 0.44 \\
\hline 14 & KK65 & $\mathrm{J} 0742+1633$ & dIrr & 279 & 8.01 & 0.14 & 15.51 & -14.15 & 15.34 & - & - & 2.45 & - & - & 0.37 \\
\hline 15 & SDSS & $\mathrm{J} 0744+2508$ & $\mathrm{Ir}$ & 749 & 12.95 & 0.18 & 18.11 & -12.63 & 18.35 & 1.38 & 0.79 & 0.30 & 0.86 & 7.21 & 0.84 \\
\hline 16 & SDSS & $\mathrm{J} 0744+2506$ & Ir & 669 & 12.95 & 0.18 & 20.47 & -10.28 & 20.30 & - & - & 0.07 & - & - & 0.83 \\
\hline 17 & MCG9-13-52 & $\mathrm{J} 0746+5117$ & $\mathrm{Sm}$ & 445 & 10.10 & 0.27 & 16.54 & -13.75 & 16.78 & 6.25 & 1.27 & 1.32 & 0.86 & 7.66 & 0.74 \\
\hline 18 & MCG9-13-56 & $\mathrm{J} 0747+5111$ & $\mathrm{Sm}$ & 439 & 10.00 & 0.30 & 15.12 & -15.18 & 15.48 & 13.69 & 0.75 & 2.46 & 0.88 & 7.70 & 0.68 \\
\hline 19 & UGC4115 & $\mathrm{J} 0757+1423$ & IAm & 341 & 7.73 & 0.12 & 14.81 & -14.75 & 15.23 & 8.37 & 0.68 & 3.69 & 0.75 & 7.60 & 0.60 \\
\hline 20 & UGC4117 & $\mathrm{J} 0757+3556$ & IBm & 773 & 14.12 & 0.20 & 15.36 & -15.59 & 15.34 & 23.73 & 0.89 & 3.57 & 0.90 & 7.82 & 0.75 \\
\hline 21 & UGC4148 & $\mathrm{J} 0800+4211$ & Scd & 716 & 13.55 & 0.18 & 15.66 & -15.18 & 15.63 & 54.15 & 2.94 & 2.95 & 0.96 & 7.83 & 0.42 \\
\hline 22 & NGC2500 & $\mathrm{J} 0801+5044$ & SBcd & 504 & 10.88 & 0.17 & 12.14 & -18.21 & 12.23 & 96.60 & 0.32 & 126.82 & 0.50 & 8.36 & 1.00 \\
\hline 23 & MCG7-17-19 & $\mathrm{J} 0809+4135$ & $\mathrm{Sc}$ & 704 & 13.37 & 0.22 & 15.46 & -15.39 & 16.65 & 25.31 & 1.14 & 5.74 & 0.85 & 7.96 & 0.60 \\
\hline 24 & SDSS & $\mathrm{J} 0810+1837$ & $\mathrm{Sm}$ & 1483 & 23.05 & 0.16 & 18.39 & -13.59 & 18.29 & 6.27 & 1.48 & 1.15 & 0.88 & 7.79 & 0.46 \\
\hline 25 & SDSS & $\mathrm{J} 0812+4836$ & $\mathrm{dIr}$ & 521 & 11.05 & 0.22 & 17.36 & -13.08 & 17.23 & 4.85 & 1.83 & 0.37 & 0.95 & 7.27 & 0.52 \\
\hline 26 & NGC2537 & $\mathrm{J} 0813+4559$ & $\mathrm{BCG}$ & 445 & 9.86 & 0.23 & 12.49 & -17.71 & 12.27 & 48.44 & 0.26 & 120.20 & 0.35 & 8.37 & 0.94 \\
\hline 27 & IC2233 & $\mathrm{J} 0813+4544$ & $\mathrm{Sd}$ & 553 & 10.70 & 0.22 & 13.34 & -17.03 & 13.05 & 54.30 & 0.54 & 25.25 & 0.74 & 8.08 & 0.11 \\
\hline 28 & NGC2541 & $\mathrm{J} 0814+4903$ & $\mathrm{SABC}$ & 548 & 12.00 & 0.22 & 12.34 & -18.28 & 12.25 & 460.82 & 1.45 & 95.03 & 0.87 & 8.02 & 0.60 \\
\hline 29 & NGC2552 & $\mathrm{J} 0819+5000$ & SAm & 524 & 11.11 & 0.20 & 13.01 & -17.42 & 12.92 & 83.01 & 0.58 & 41.66 & 0.73 & 8.28 & 0.68 \\
\hline 30 & KUG0821+321 & $\mathrm{J} 0825+3201$ & Ir & 648 & 12.25 & 0.20 & 16.91 & -13.73 & 16.10 & 3.54 & 0.73 & 1.27 & 0.79 & 7.60 & 0.67 \\
\hline 31 & HS0822+3542 & $\mathrm{J} 0825+3532$ & BCG & 720 & 13.49 & 0.20 & 17.88 & -12.97 & 17.92 & 1.46 & 0.61 & 0.04 & 0.98 & 7.44 & 0.78 \\
\hline 32 & $\mathrm{SAO} 0822+3545$ & $\mathrm{~J} 0826+3535$ & $\operatorname{Im}$ & 740 & 13.49 & 0.20 & 17.74 & -13.11 & 17.56 & 4.30 & 1.57 & 0.16 & 0.97 & - & 0.80 \\
\hline 33 & UGC4426 & $\mathrm{J} 0828+4151$ & Im & 397 & 10.30 & 0.16 & 15.35 & -14.87 & 15.13 & 27.05 & 1.96 & 6.18 & 0.85 & 7.58 & 0.63 \\
\hline
\end{tabular}


Table 1. Basic parameters of the galaxies in the Lynx?Cancer void. (Condt.)

\begin{tabular}{|c|c|c|c|c|c|c|c|c|c|c|c|c|c|c|c|}
\hline & $\begin{array}{l}\text { Name or } \\
\text { prefix } \\
(1)\end{array}$ & $\left|\begin{array}{c}\text { Name in brief } \\
\text { IAU format } \\
(2)\end{array}\right|$ & $\left|\begin{array}{c}\text { Type } \\
(3)\end{array}\right|$ & \begin{tabular}{|c|}
$V_{\text {hel }}$ \\
$\mathrm{km} / \mathrm{s}$ \\
$(4)$
\end{tabular} & $\left|\begin{array}{c}\text { Dist } \\
\mathrm{Mpc} \\
(5)\end{array}\right|$ & $\begin{array}{c}A_{\mathrm{B}}^{\mathrm{NED}} \\
\text { mag } \\
(6)\end{array}$ & \begin{tabular}{|c}
$B_{\text {tot }}$ \\
mag \\
$(7)$
\end{tabular} & $\left|\begin{array}{c}M_{\mathrm{B}, 0} \\
\mathrm{mag} \\
(8)\end{array}\right|$ & $\left|\begin{array}{c}B_{\mathrm{tot}}^{\mathrm{NED}} \\
\mathrm{mag} \\
(9)\end{array}\right|$ & $\mid \begin{array}{c}\mathfrak{M}(\mathrm{HI}) \\
10^{7} M_{\odot} \\
(10)\end{array}$ & $\left|\begin{array}{c}\mathfrak{M}(\mathrm{HI}) / L_{\mathrm{B}} \\
(11)\end{array}\right|$ & $\left|\begin{array}{r}\mathfrak{M}_{\text {star }} \\
10^{7} M_{\odot} \\
(12)\end{array}\right|$ & $\left|\begin{array}{l}f_{\text {gas }} \\
(13)\end{array}\right|$ & $\left|\begin{array}{c}12+\log (\mathrm{O} / \mathrm{H}) \\
(14)\end{array}\right|$ & $\mid \begin{array}{l}\mathrm{b} / \mathrm{a} \\
(15)\end{array}$ \\
\hline 34 & SDSS & $\mathrm{J} 0831+4104$ & |LSB & 582 & | 11.64 & 0.16 & |17.71 & $\mid-12.78$ & 17.71 & 0.58 & 0.29 & 0.59 & |0.57| & - & 0.90 \\
\hline 35 & DSS & $\mathrm{J} 0843+4025$ & Im & 614 & 12.05 & 0.14 & 17.90 & $-12.65 \mid$ & 17.83 & 0.62 & 0.35 & 0.41 & 0.67 & 7.49 & 0.70 \\
\hline 36 & SDSS & $\mathrm{J} 0845+1519$ & dI & 1642 & 24.99 & 0.09 & 18.61 & -13.47 & 18.60 & & & 2.14 & & - & 0.79 \\
\hline 37 & SDSS & $\mathrm{J} 0852+1350$ & Im & 1511 & 23.08 & 0.16 & 17.40 & -14.58 & 17.43 & 26.40 & 2.50 & 2.98 & 0.92 & 7.28 & 0.76 \\
\hline 38 & SDSS & $\mathrm{J} 0852+1351$ & dI & 1541 & 23.08 & 0.16 & 19.82 & -12.16 & 19.73 & & & 0.22 & & - & 0.64 \\
\hline 39 & UGC4704 & $\mathrm{J} 0859+3912$ & $\mathrm{Sdm}$ & 596 & 11.74 & 0.13 & 14.82 & $-15.66 \mid$ & 15.51 & 72.86 & 2.56 & 6.73 & 0.94 & 7.96 & 0.13 \\
\hline 40 & SDSS & J0859+3923 & dI & 588 & 11.63 & 0.11 & 17.25 & -13.19 & 16.98 & 1.56 & 0.53 & 0.96 & 0.69 & 7.57 & 0.93 \\
\hline 41 & SDSS & $\mathrm{J} 0900+3222$ & dI & 740 & 13.45 & 0.14 & 18.97 & -11.81 & 18.86 & - & & 0.42 & & - & 0.64 \\
\hline 42 & UGC4722 & $\mathrm{J} 0900+2536$ & $\mathrm{Sdm}$ & 1794 & 27.89 & 0.17 & 14.86 & -17.54 & 15.16 & - & & 22.59 & & 7.66 & 0.18 \\
\hline 43 & SDSS & $\mathrm{J} 0900+4908$ & $\mathrm{Sdm}$ & 1600 & 25.78 & 0.09 & 18.49 & $-13.65 \mid$ & 18.63 & - & & 1.45 & - & - & 0.72 \\
\hline 44 & SDSS & J0908+0657 & $\mathrm{Sdm}$ & 1571 & 23.45 & 0.19 & 17.06 & -14.98 & 17.00 & 4.80 & 0.31 & 5.50 & 0.54 & - & 0.57 \\
\hline 45 & SDSS & J0908+0517 & dI & 598 & 10.01 & 0.20 & 17.21 & -12.99 & 16.96 & 2.70 & 1.10 & 0.02 & 0.95 & 7.60 & 0.99 \\
\hline 46 & SDSS & J0911+3135 & dI & 750 & 13.52 & 0.07 & 18.05 & $-12.68 \mid$ & 17.97 & 1.81 & 0.99 & 0.24 & 0.91 & 7.50 & 0.87 \\
\hline 47 & IC2450 & $\mathrm{J} 0917+2525$ & S0 & 1644 & 25.47 & 0.14 & 14.06 & -18.11 & 13.84 & & & 185.06 & & - & 0.61 \\
\hline 48 & SDSS & J0926+3343 & LSB & 536 & 10.63 & 0.08 & 17.30 & -12.91 & 17.34 & 6.77 & 2.98 & 0.13 & 0.99 & 7.12 & 0.25 \\
\hline 49 & SDSS & J0928+2845 & dI & 1229 & 19.90 & 0.09 & 16.76 & -14.82 & 16.70 & 23.37 & 1.77 & 8.32 & 0.79 & 7.68 & 0.49 \\
\hline 50 & SDSS & $\mathrm{J} 0929+2502$ & $\mathrm{dI}$ & 1661 & 25.66 & 0.14 & 19.24 & -12.95 & 19.03 & 5.59 & 2.38 & 0.33 & 0.96 & 7.20 & 0.89 \\
\hline 51 & SDSS & J0929+1155 & $\mathrm{dI}$ & 1614 & 24.29 & 0.11 & 17.20 & $-14.84 \mid$ & 17.36 & - & & 2.76 & & - & 0.40 \\
\hline 52 & SDSS & $\mathrm{J} 0931+2717$ & $\mathrm{Sm}$ & 1505 & 23.60 & 0.08 & 18.00 & $-13.94 \mid$ & 17.98 & - & & 1.06 & & 7.55 & 0.52 \\
\hline 53 & CGCG035-007 & J0934+0625 & $\mathrm{Sc}$ & 574 & 9.23 & 0.18 & 15.42 & $|-14.59|$ & 15.22 & 12.67 & 1.19 & 5.22 & 0.76 & - & 0.78 \\
\hline 54 & KUG0934+277 & J0937+2733 & $\operatorname{Im}$ & 1594 & 25.16 & 0.08 & 16.50 & $-15.59 \mid$ & 16.53 & & & 7.35 & & - & 0.74 \\
\hline 55 & SDSS & J0940+4459 & dI & 1246 & 20.71 & 0.06 & 18.01 & $-13.63 \mid$ & 17.96 & & & 1.60 & & 7.40 & 0.72 \\
\hline 56 & KISSB23 & $\mathrm{J} 0940+2935$ & $\mathrm{Sd}$ & 505 & 10.21 & 0.10 & 16.07 & -14.08 & 16.32 & 5.41 & 0.81 & 1.29 & 0.85 & 7.60 & 0.49 \\
\hline 57 & SDSS & J0942+0937 & dI & 1461 & 22.00 & 0.11 & 18.15 & -13.67 & 21.40 & & & 2.31 & & - & 0.81 \\
\hline 58 & UGC5186 & J0942+3315 & Im & 551 & 10.77 & 0.06 & 15.99 & -14.23 & 16.27 & 2.57 & 0.34 & 2.08 & 0.62 & - & 0.40 \\
\hline 59 & SDSS & J0943+4134 & dI & 1403 & 22.77 & 0.06 & 17.64 & -14.21 & 17.63 & 4.89 & 0.65 & 2.47 & 0.73 & - & 0.56 \\
\hline 60 & SDSS & J0944+1000 & dI & 1477 & 22.22 & 0.12 & 16.96 & $-14.90 \mid$ & 16.95 & 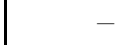 & & 3.88 & & - & 0.90 \\
\hline 61 & IC559 & J0944+0936 & $\mathrm{Sc}$ & 541 & 9.40 & 0.12 & 14.77 & $-15.22 \mid$ & 14.98 & & & 6.33 & & - & 0.87 \\
\hline 62 & UGC5209 & J0945+3214 & Im & 538 & 10.55 & 0.08 & 16.32 & $|-13.88|$ & 16.06 & 4.02 & 0.72 & 1.87 & 0.74 & - & 0.75 \\
\hline 63 & SDSS & J0947+4138 & dI & 1389 & 22.56 & 0.07 & 17.92 & -13.92 & 17.61 & 1.20 & 0.21 & 0.46 & 0.78 & 7.76 & 0.54 \\
\hline 64 & MRK407 & J0947+3905 & comp & 1589 & 25.21 & 0.07 & 15.28 & $-16.80 \mid$ & 15.27 & - & & 17.66 & - & - & 0.74 \\
\hline 65 & UZCJ09475+390 & & dI & 1553 & 24.73 & 0.07 & 16.85 & -15.19 & 17.00 & - & - & 3.15 & & 7.50 & 0.63 \\
\hline 66 & SDSS & $\mathrm{J} 0947+3905$ & dI & 1479 & 23.70 & 0.07 & 18.03 & $-13.92 \mid$ & 18.12 & & & 1.41 & & - & 0.63 \\
\hline
\end{tabular}


Table 1. Basic parameters of the galaxies in the Lynx?Cancer void. (Condt.)

\begin{tabular}{|c|c|c|c|c|c|c|c|c|c|c|c|c|c|c|c|}
\hline & $\begin{array}{l}\text { Name or } \\
\text { prefix } \\
(1)\end{array}$ & $\begin{array}{l}\text { Name in brief } \\
\text { IAU format } \\
(2)\end{array}$ & $\begin{array}{c}\text { Type } \\
(3)\end{array}$ & $\begin{array}{c}V_{\text {hel }} \\
\mathrm{km} / \mathrm{s} \\
(4)\end{array}$ & $\left|\begin{array}{c}\text { Dist } \\
\mathrm{Mpc} \\
(5)\end{array}\right|$ & $\begin{array}{c}A_{\mathrm{B}}^{\mathrm{NED}} \\
\operatorname{mag} \\
(6)\end{array}$ & $\left|\begin{array}{c}B_{\text {tot }} \\
\text { mag } \\
(7)\end{array}\right|$ & $\left|\begin{array}{c}M_{\mathrm{B}, 0} \\
\mathrm{mag} \\
(8)\end{array}\right|$ & $\left|\begin{array}{c}B_{\text {tot }}^{\text {NED }} \\
\text { mag } \\
(9)\end{array}\right|$ & $\left|\begin{array}{c}\mathfrak{M}(\mathrm{HI}) \\
10^{7} M_{\odot} \\
(10)\end{array}\right|$ & $\mid \begin{array}{c}\mathfrak{M}(\mathrm{HI}) / L_{\mathrm{B}} \\
(11)\end{array}$ & $\mid \begin{array}{r}\mathfrak{M}_{\text {star }} \\
10^{7} M_{\odot} \\
(12)\end{array}$ & $\begin{array}{l}f_{\text {gas }} \\
(13)\end{array}$ & $\begin{array}{c}12+\log (\mathrm{O} / \mathrm{H}) \\
(14)\end{array}$ & $\left|\begin{array}{l}\mathrm{b} / \mathrm{a} \\
(15)\end{array}\right|$ \\
\hline 67 & UGC5272b & $\mathrm{J} 0950+3127$ & Im & 539 & $\mid 10.27$ & 0.10 & $|17.56|$ & $|-12.60|$ & 17.68 & 2.89 & 1.70 & 0.19 & | $0.95 \mid$ & 7.60 & 0.67 \\
\hline 68 & UGC5272 & $\mathrm{J} 0950+3129$ & $\operatorname{Im}$ & 520 & 10.30 & 0.10 & 14.45 & -15.71 & 14.46 & 46.33 & 1.55 & 3.41 & 0.95 & 7.84 & 0.42 \\
\hline 69 & UGC5288 & J0951+0749 & $\mathrm{Sdm}$ & 556 & 9.47 & 0.15 & 14.42 & -15.61 & 14.62 & - & - & 9.69 & - & - & 0.92 \\
\hline 70 & SDSS & $\mathrm{J} 0951+3842$ & $\mathrm{dI}$ & 1435 & 23.07 & 0.08 & 17.46 & -14.44 & 17.42 & - & - & 1.77 & - & 7.85 & 0.89 \\
\hline 71 & SDSS & $\mathrm{J} 0954+3620$ & $\mathrm{dI}$ & 503 & 10.22 & 0.04 & 18.05 & -12.04 & 17.93 & 0.62 & 0.60 & 0.16 & 0.84 & - & 0.62 \\
\hline 72 & IC 2520 & $\mathrm{~J} 0956+2713$ & $\mathrm{~S}$ & 1243 & 19.93 & 0.09 & 14.27 & -17.32 & 14.70 & - & - & 141.81 & - & - & 0.87 \\
\hline 73 & SDSS & $\mathrm{J} 0956+2716$ & $\mathrm{dI}$ & 1074 & 17.63 & 0.09 & 18.13 & -13.19 & 18.39 & - & - & 0.75 & - & - & 0.54 \\
\hline 74 & UGC5340 & $\mathrm{J} 0956+2849$ & $\mathrm{BC} / \mathrm{Im}$ & 502 & 9.86 & 0.08 & 14.70 & -15.35 & 14.60 & 66.35 & 3.10 & 1.50 & 0.98 & 7.14 & 0.44 \\
\hline 75 & SDSS & $\mathrm{J} 0957+2745$ & $\mathrm{dI}$ & 1184 & 19.14 & 0.08 & 18.16 & -13.33 & 18.08 & - & - & 0.63 & - & - & 0.60 \\
\hline 76 & UGC5354 & $\mathrm{J} 0958+4744$ & $\mathrm{Sm}$ & 1168 & 19.68 & 0.07 & 14.15 & -17.39 & 14.60 & - & - & 33.42 & - & - & 0.56 \\
\hline 77 & $\mathrm{PC} 0956+4751$ & $\mathrm{~J} 0959+4736$ & $\mathrm{dI}$ & 1093 & 18.66 & 0.07 & 17.05 & -14.37 & 17.14 & 14.87 & 1.70 & 1.91 & 0.91 & - & 0.45 \\
\hline 78 & SDSS & $\mathrm{J} 1000+3032$ & $\mathrm{dI}$ & 501 & 9.90 & 0.08 & 18.14 & -11.92 & 18.06 & 0.28 & 0.30 & 0.32 & 0.53 & 7.42 & 0.79 \\
\hline 79 & KUG0959+299 & $\mathrm{J} 1002+2943$ & $\mathrm{dI}$ & 766 & 13.48 & 0.10 & 17.36 & -13.39 & 17.32 & - & - & 0.58 & - & - & 0.77 \\
\hline 80 & UGC5427 & $\mathrm{J} 1004+2921$ & SABd & 498 & 9.79 & 0.10 & 14.91 & -15.15 & 14.89 & 5.98 & 0.34 & 5.20 & 0.60 & 7.90 & 0.64 \\
\hline 81 & UGC5464 & $\mathrm{J} 1008+2932$ & $\mathrm{Sm}$ & 1003 & 16.90 & 0.10 & 15.77 & -15.47 & 15.62 & 19.29 & 0.81 & 9.58 & 0.73 & 7.86 & 0.46 \\
\hline 82 & SDSS & $\mathrm{J} 1010+4617$ & $\mathrm{dI}$ & 1092 & 18.58 & 0.03 & 18.23 & -13.15 & 18.20 & 0.49 & 0.17 & 0.57 & 0.53 & 7.78 & 0.45 \\
\hline 83 & UGC5540 & $\mathrm{J} 1016+3746$ & $\mathrm{Sc}$ & 1162 & 19.16 & 0.07 & 14.63 & -16.85 & 14.60 & 46.55 & 0.54 & 26.89 & 0.70 & 8.00 & 0.36 \\
\hline 84 & HS1013+3809 & $\mathrm{J} 1016+3754$ & $\mathrm{BCG}$ & 1173 & 19.30 & 0.07 & 16.02 & -15.48 & 15.99 & 13.28 & 0.55 & 0.33 & 0.98 & 7.59 & 0.65 \\
\hline 85 & SDSS & $\mathrm{J} 1019+2923$ & $\mathrm{dI}$ & 874 & 15.25 & 0.14 & 17.48 & $\mid-13.57$ & 17.43 & 8.23 & 1.97 & 0.60 & 0.95 & - & 0.41 \\
\hline
\end{tabular}


Table 2. Photometric paramerets I.

\begin{tabular}{|c|c|c|c|c|c|c|c|c|c|c|c|}
\hline & $\begin{array}{l}\text { Name } \\
\text { (1) }\end{array}$ & $\left|\begin{array}{c}R_{50}(g) \\
\prime \prime \\
(2)\end{array}\right|$ & $\left|\begin{array}{c}R_{90}(g) \\
\prime \\
(3)\end{array}\right|$ & $\left|\begin{array}{c}\mu_{\mathrm{eff}}(g) \\
m / \square^{\prime \prime} \\
(4)\end{array}\right|$ & $\left|\begin{array}{c}R^{25}(g) \\
\prime \prime \\
(5)\end{array}\right|$ & $\begin{array}{l}a_{25} \\
\operatorname{kpc} \\
(6)\end{array} \mid$ & $\begin{array}{c}g^{25} \\
\operatorname{mag} \\
(7)\end{array}$ & $\left|\begin{array}{c}R^{26.5}(g) \\
\prime \prime\end{array}\right|$ & $\left|\begin{array}{c}g^{26.5} \\
\operatorname{mag} \\
(9)\end{array}\right|$ & $\begin{array}{c}\text { Opt.size } \\
\quad \text { " } \\
(10)\end{array}$ & $\begin{array}{c}\text { Opt.size } \\
\text { kpc } \\
(11)\end{array}$ \\
\hline 1 & HIPASSJ0626+24| & 9.9 & 18.5 & 24.7 & $13.7 \mid$ & 1.5 & $|17.9|$ & 20.6 & $|17.6|$ & $13.7 \times 8.1$ & $1.5 \times 0.9$ \\
\hline 2 & UGC3600 & 26.9 & 75.9 & 24.0 & 40.0 & 1.8 & \begin{tabular}{l|l}
36.1 & 16.1 \\
\end{tabular} & 74.4 & $\mid 15.7$ & $40.0 \times 10.8$ & $1.8 \times 0.5$ \\
\hline 3 & UGC3672 & 18.2 & 43.3 & 24.1 & 27.7 & 2.3 & 16.1 & 47.0 & 15.8 & $27.7 \times 15.2$ & $2.3 \times 1.2$ \\
\hline 4 & $\mathrm{~J} 0713+2926$ & 11.8 & 25.4 & 23.9 & 20.7 & 1.6 & 16.6 & 27.0 & 16.4 & $20.7 \times 13.1$ & $1.6 \times 1.0$ \\
\hline 5 & $\mathrm{~J} 0723+3621$ & 15.3 & 35.6 & 24.1 & 24.9 & 1.9 & 17.0 & 39.2 & $\mid 16.7$ & $24.9 \times 7.8$ & $1.9 \times 0.6$ \\
\hline 6 & $\mathrm{~J} 0723+3622$ & 3.8 & 7.7 & 24.5 & 5.5 & 0.4 & 19.7 & 9.1 & 19.3 & $5.5 \times 3.9$ & $0.4 \times 0.3$ \\
\hline 7 & $\mathrm{~J} 0723+3624$ & 3.9 & 6.9 & 26.3 & 1.8 & 0.1 & 22.7 & 5.5 & 21.3 & $1.8 \times 0.9$ & $0.1 \times 0.1$ \\
\hline 8 & UGC3860 & 27.1 & 56.3 & 24.2 & 42.5 & 1.6 & 15.1 & 56.1 & $\mid 15.0$ & $42.5 \times 24.0$ & $1.6 \times 0.9$ \\
\hline 9 & UGC3876 & 34.0 & 68.6 & 23.2 & 71.2 & 5.2 & 13.5 & 91.3 & 13.4 & $71.2 \times 41.5$ & $5.2 \times 3.0$ \\
\hline 10 & J0730+4109 & 5.3 & 14.1 & 22.1 & 14.9 & 1.1 & 16.4 & 21.3 & 16.4 & $14.9 \times 8.8$ & $1.1 \times 0.7$ \\
\hline 11 & J0737+4724 & 7.3 & 18.7 & 24.0 & 12.0 & 0.6 & 18.1 & 19.6 & $\mid 17.9$ & $12.0 \times 5.7$ & $0.6 \times 0.3$ \\
\hline 12 & UGC3966 & 26.8 & 46.7 & 24.8 & 38.2 & 1.6 & 15.4 & 52.3 & 15.1 & $38.2 \times 33.0$ & $1.6 \times 1.4$ \\
\hline 13 & DDO47 & 52.0 & 106.4 & 25.0 & 60.2 & 2.3 & 15.1 & 103.5 & $\mid 14.7$ & $60.2 \times 26.5$ & $2.4 \times 1.0$ \\
\hline 14 & KK65 & 22.1 & 53.1 & 23.6 & 41.5 & 1.6 & 15.4 & 63.9 & 15.2 & $41.5 \times 15.2$ & $1.6 \times 0.6$ \\
\hline 15 & $\mathrm{~J} 0744+2508$ & 2.7 & 6.8 & 22.5 & 7.7 & 0.5 & 17.9 & 10.0 & 17.8 & $7.7 \times 6.5$ & $0.5 \times 0.4$ \\
\hline 16 & J0744+2506 & 1.6 & 4.0 & 23.6 & 3.2 & 0.2 & 20.4 & 4.7 & 20.2 & $3.2 \times 2.6$ & $0.2 \times 0.2$ \\
\hline 17 & MCG9-13-52 & 15.1 & 29.1 & 24.5 & 21.6 & 1.1 & 16.5 & 32.3 & 16.3 & $21.6 \times 16.0$ & $1.1 \times 0.8$ \\
\hline 18 & MCG9-13-56 & 7.9 & 20.5 & 21.6 & 25.6 & 1.2 & 14.9 & 32.8 & 14.8 & $25.6 \times 17.4$ & $1.2 \times 0.8$ \\
\hline 19 & UGC4115 & 27.6 & 59.0 & 23.9 & 49.4 & 1.8 & 14.6 & 72.1 & 14.4 & $49.4 \times 29.5$ & $1.9 \times 1.1$ \\
\hline 20 & UGC4117 & 15.7 & 34.0 & 23.5 & 30.6 & 2.1 & 15.2 & 41.6 & 15.1 & $30.6 \times 22.9$ & $2.1 \times 1.6$ \\
\hline 21 & UGC4148 & 22.9 & 70.6 & 24.0 & 33.2 & 2.2 & 15.9 & 65.6 & 15.5 & $33.2 \times 13.9$ & $2.2 \times 0.9$ \\
\hline 22 & NGC2500 & 43.9 & 82.1 & 22.7 & 92.8 & 4.9 & 11.9 & 117.0 & 11.8 & $92.8 \times 92.5$ & $4.9 \times 4.9$ \\
\hline 23 & MCG71719 & 19.6 & 38.0 & 23.8 & 32.7 & 2.1 & 15.3 & 44.8 & 15.2 & $32.7 \times 19.4$ & $2.1 \times 1.3$ \\
\hline 24 & J0810+1837 & 5.5 & 15.6 & 23.6 & 10.4 & 1.2 & 18.3 & 15.5 & $\mid 18.2$ & $10.4 \times 4.8$ & $1.2 \times 0.5$ \\
\hline 25 & J0812+4836 & 6.1 & 14.3 & 23.0 & 14.0 & 0.8 & 17.2 & 20.0 & 17.1 & $14.0 \times 7.3$ & $0.8 \times 0.4$ \\
\hline 26 & NGC2537 & 20.0 & 44.8 & 21.3 & 64.6 & 3.1 & 12.1 & 78.1 & 12.1 & $64.6 \times 60.8$ & $3.1 \times 2.9$ \\
\hline 27 & IC2233 & 125.0 & 320.3 & 23.8 & 212.7 & 11.0 & 13.4 & 380.5 & $\mid 13.0$ & $212.7 \times 22.1$ & $11.0 \times 1.1$ \\
\hline 28 & NGC2541 & 70.5 & 155.0 & 23.4 & 143.2 & 8.3 & 12.1 & 186.7 & 12.1 & $143.2 \times 85.9$ & $8.3 \times 5.0$ \\
\hline 29 & NGC2552 & 51.8 & 96.5 & 23.6 & 97.9 & 5.3 & 12.8 & 123.2 & $|12.7|$ & $97.9 \times 66.2$ & $5.3 \times 3.6$ \\
\hline 30 & KUG0821+321 & 6.1 & 15.3 & 22.8 & 15.1 & 0.9 & 16.7 & 20.5 & $|16.6|$ & $15.1 \times 10.1$ & $0.9 \times 0.6$ \\
\hline 31 & HS0822+3542 & 1.1 & 5.4 & 20.4 & 6.4 & 0.4 & 17.7 & 9.7 & $|17.7|$ & $6.4 \times 5.0$ & $0.4 \times 0.3$ \\
\hline 32 & $\mathrm{SAO} 0822+3545$ & 5.8 & 12.3 & 23.8 & 10.7 & 0.7 & 17.7 & 15.1 & 17.5 & $10.7 \times 8.6$ & $0.7 \times 0.6$ \\
\hline 33 & UGC4426 & 36.5 & 72.0 & 25.1 & 37.3 & 1.9 & 15.8 & 69.4 & 15.1 & $37.3 \times 23.4$ & $1.9 \times 1.2$ \\
\hline 34 & J0831+4104 & 3.5 & 10.6 & 22.7 & 8.7 & 0.5 & 17.5 & 13.0 & $\mid 17.4$ & $8.7 \times 7.8$ & $0.5 \times 0.4$ \\
\hline 35 & $\mathrm{~J} 0843+4025$ & 3.2 & 8.8 & 22.4 & 9.0 & 0.5 & 17.7 & 12.9 & 17.6 & $9.0 \times 6.3$ & $0.5 \times 0.4$ \\
\hline 36 & J0845+1519 & 3.5 & 9.8 & 23.4 & 7.0 & 0.8 & 18.5 & 11.5 & $\mid 18.3$ & $7.0 \times 5.5$ & $0.8 \times 0.7$ \\
\hline 37 & J0852+1350 & 8.1 & 17.8 & 24.1 & 13.4 & 1.5 & 17.4 & 20.6 & 17.1 & $13.4 \times 10.3$ & $1.5 \times 1.1$ \\
\hline 38 & $\mathrm{~J} 0852+1351$ & 1.2 & 4.9 & 22.2 & 4.4 & 0.5 & 19.6 & 6.4 & $\mid 19.5$ & $4.4 \times 2.8$ & $0.5 \times 0.3$ \\
\hline 39 & UGC4704 & 83.8 & 235.9 & 24.6 & 87.8 & 5.0 & 15.1 & 200.5 & 14.6 & $87.8 \times 11.4$ & $5.0 \times 0.7$ \\
\hline 40 & J0859+3923 & 5.6 & 16.5 & 23.3 & 11.3 & 0.6 & 17.2 & 19.5 & 17.0 & $11.3 \times 10.5$ & $0.6 \times 0.6$ \\
\hline 41 & $\mathrm{~J} 0900+3222$ & 4.6 & 10.2 & 24.2 & 7.2 & 0.5 & 18.9 & 11.1 & 18.7 & $7.2 \times 4.6$ & $0.5 \times 0.3$ \\
\hline 42 & UGC4722 & 45.0 & 163.1 & 23.7 & 76.1 & 10.3 & 14.9 & 122.8 & 14.7 & $76.1 \times 13.3$ & $10.3 \times 1.8$ \\
\hline 43 & J0900+4908 & 6.6 & 15.2 & 24.7 & 8.1 & $\mid 1.0$ & 18.7 & 14.0 & 18.3 & $8.1 \times 5.8$ & $1.0 \times 0.7$ \\
\hline 44 & J0908+0657 & 8.3 & 21.0 & 23.4 & 15.9 & 1.8 & 16.9 & 24.3 & 16.8 & $15.9 \times 9.0$ & $1.8 \times 1.0$ \\
\hline 45 & J0908+0517 & 11.9 & 20.8 & 25.0 & 13.7 & 0.7 & 17.5 & 22.8 & 17.0 & $13.7 \times 13.5$ & $0.7 \times 0.7$ \\
\hline 46 & J0911+3135 & 2.1 & 5.3 & 22.0 & 6.6 & 0.4 & 17.8 & 9.1 & 17.8 & $6.6 \times 5.8$ & $0.4 \times 0.4$ \\
\hline 47 & IC2450 & 13.0 & 39.7 & 21.4 & 45.0 & 5.6 & 13.8 & 62.6 & 13.7 & $45.0 \times 27.2$ & $5.6 \times 3.4$ \\
\hline 48 & J0926+3343 & 22.1 & 47.5 & 25.0 & 21.5 & 1.1 & $\mid 17.8$ & 47.9 & 17.2 & $21.5 \times 5.4$ & $1.1 \times 0.3$ \\
\hline 49 & $\mathrm{~J} 0928+2845$ & 5.8 & 16.5 & 22.2 & 16.8 & 1.6 & $|16.5|$ & 24.7 & $|16.4|$ & $16.8 \times 8.3$ & $1.6 \times 0.8$ \\
\hline
\end{tabular}


Table 2. Photometric paramerets I. (Condt.)

\begin{tabular}{|c|c|c|c|c|c|c|c|c|c|c|c|}
\hline & Name & $\left|\begin{array}{c}R_{50}(g) \\
\prime \prime \\
(2)\end{array}\right|$ & $\left|\begin{array}{c}R_{90}(g) \\
\prime \\
(3)\end{array}\right|$ & $\mid \begin{array}{c}\mu_{\mathrm{eff}}(g) \\
m / \square^{\prime \prime} \\
(4)\end{array}$ & $\left|\begin{array}{c}R^{25}(g) \\
\prime \prime \\
(5)\end{array}\right|$ & $\left|\begin{array}{l}a_{25} \\
\text { kpc } \\
(6)\end{array}\right|$ & $\left|\begin{array}{c}g^{25} \\
\mathrm{mag} \\
(7)\end{array}\right|$ & $\left|\begin{array}{c}R^{26.5}(g) \\
\prime \prime \\
(8)\end{array}\right|$ & $\left|\begin{array}{c}g^{26.5} \\
\text { mag } \\
(9)\end{array}\right|$ & $\begin{array}{c}\text { Opt.size } \\
\quad \text { " }\end{array}$ & $\begin{array}{c}\text { Opt.size } \\
\text { kpc } \\
(11)\end{array}$ \\
\hline 50 & J0929+2502 & 5.5 & 11.0 & 25.3 & 5.3 & |0.7| & |19.7| - n & 10.0 & $|19.1|$ & $5.3 \times 4.7$ & $0.7 \times 0.6 \mid$ \\
\hline 51 & J0929+1155 & 12.6 & 30.6 & 24.1 & 19.5 & 2.3 & 17.3 & 31.4 & 17.0 & $19.5 \times 7.8$ & $2.3 \times 0.9$ \\
\hline 52 & $\mathrm{~J} 0931+2717$ & 6.2 & 16.8 & 23.7 & 11.0 & 1.3 & 18.0 & 18.2 & 17.8 & $11.0 \times 5.8$ & $1.3 \times 0.7$ \\
\hline 53 & CGCG035-007 & 9.1 & 23.0 & 22.3 & 23.6 & 1.1 & 15.1 & 34.3 & 15.1 & $23.6 \times 18.3$ & $1.1 \times 0.8$ \\
\hline 54 & KUG0934+277 & 11.1 & 24.1 & 23.8 & 20.4 & 2.5 & 16.4 & 29.6 & 16.2 & $20.4 \times 15.2$ & $2.5 \times 1.9$ \\
\hline 55 & J0940+4459 & 2.4 & 7.1 & 21.9 & 7.8 & 0.8 & 17.8 & 10.7 & 17.7 & $7.8 \times 5.7$ & $0.8 \times 0.6$ \\
\hline 56 & KISSB23 & 9.0 & 29.7 & 22.5 & 23.6 & 1.2 & $16.0 \mid$ & 36.4 & 15.8 & $23.6 \times 11.5$ & $1.2 \times 0.6$ \\
\hline 57 & J0942+0937 & 8.8 & 17.0 & 25.0 & 9.7 & 1.0 & 18.4 & 16.8 & 17.9 & $9.7 \times 7.9$ & $1.0 \times 0.8$ \\
\hline 58 & UGC5186 & 14.6 & 46.6 & 23.2 & 30.2 & $\mid 1.6$ & 15.8 & 50.2 & $\mid 15.6$ & $30.2 \times 12.1$ & $1.6 \times 0.6$ \\
\hline 59 & J0943+4134 & 5.2 & 16.1 & 23.0 & 12.1 & 1.3 & 17.5 & 16.3 & 17.4 & $12.1 \times 6.8$ & $1.3 \times 0.8$ \\
\hline 60 & J0944+1000 & 10.6 & 20.9 & 24.4 & 16.4 & 1.8 & $\mid 16.9$ & 24.3 & $\mid 16.7$ & $16.4 \times 14.8$ & $1.8 \times 1.6$ \\
\hline 61 & IC559 & 13.6 & 43.6 & 22.7 & 35.7 & $\mid 1.6$ & 14.3 & 58.0 & $\mid 14.2$ & $35.7 \times 31.2$ & $1.6 \times 1.4$ \\
\hline 62 & UGC5209 & 15.4 & 31.9 & 24.4 & 22.7 & 1.2 & 16.3 & 29.8 & 16.1 & $22.7 \times 17.1$ & $1.2 \times 0.9$ \\
\hline 63 & J0947+4138 & 2.0 & 9.6 & 21.2 & 8.7 & 1.0 & 17.8 & 12.9 & 17.7 & $8.7 \times 4.7$ & $1.0 \times 0.5$ \\
\hline 64 & MRK407 & 2.6 & 10.2 & 19.5 & 15.8 & $\mid 1.9$ & 15.0 & 23.7 & 15.0 & $15.8 \mathrm{z}$ & $1.9 \times 1.4$ \\
\hline 65 & J0947+3908 & 7.8 & 15.6 & 23.2 & 16.5 & 2.0 & 16.6 & 21.5 & 16.6 & $16.5 \times 10.3$ & $2.0 \times 1.2$ \\
\hline 66 & J0947+3905 & 4.9 & 19.2 & 23.4 & 8.5 & 1.0 & 18.1 & 15.9 & 17.9 & $8.5 \times 5.4$ & $1.0 \times 0.6$ \\
\hline 67 & UGC5272b & 5.1 & 12.8 & 23.1 & 11.0 & 0.5 & 17.5 & 15.8 & $\mid 17.4$ & $11.0 \times 7.3$ & $0.5 \times 0.4$ \\
\hline 68 & UGC5272 & 32.5 & 73.8 & 23.5 & 66.7 & 3.3 & 14.4 & 86.2 & 14.2 & $66.7 \times 28.1$ & $3.3 \times 1.4$ \\
\hline 69 & UGC5288 & 15.5 & 42.6 & 22.7 & 38.6 & 1.8 & 14.2 & 57.6 & $\mid 14.1$ & $38.6 \times 35.4$ & $1.8 \times 1.6$ \\
\hline 70 & |J0951+3842 & 6.2 & 15.4 & 23.8 & 11.2 & 1.3 & 17.4 & 16.7 & 17.2 & $11.2 \times 10.0$ & $1.3 \times 1.1$ \\
\hline 71 & J0954+3620 & 4.6 & 12.5 & 23.3 & 9.7 & 0.5 & $|18.0|$ & 14.8 & 17.8 & $9.7 \times 6.0$ & $0.5 \times 0.3$ \\
\hline 72 & IC2520 & 8.5 & 17.3 & 21.1 & 25.7 & 2.5 & 13.8 & 31.4 & 13.8 & $25.7 \times 22.3$ & $2.5 \times 2.2$ \\
\hline 73 & J0956+2716 & 10.8 & 22.9 & 25.1 & 11.4 & 1.0 & 18.5 & 22.0 & 18.0 & $11.4 \times 6.1$ & $1.0 \times 0.5$ \\
\hline 74 & UGC5340 & 31.3 & 82.4 & 23.8 & 55.6 & 2.7 & 14.8 & 80.7 & 14.6 & $55.6 \times 24.7$ & $2.7 \times 1.2$ \\
\hline 75 & J0957+2745 & 4.4 & 10.2 & 23.3 & 9.3 & 0.9 & 18.0 & 13.3 & $\mid 17.9$ & $9.3 \times 5.6$ & $0.9 \times 0.5$ \\
\hline 76 & UGC5354 & 25.6 & 53.6 & 23.0 & 53.8 & 5.1 & 13.9 & 74.4 & 13.9 & $53.8 \times 30.4$ & $=5.1 \times 2.9$ \\
\hline 77 & $\mathrm{PC} 0956+4751$ & 9.3 & 25.1 & 23.4 & 18.3 & 1.7 & 17.0 & 29.0 & 16.8 & $18.3 \times 8.3$ & $1.7 \times 0.8$ \\
\hline 78 & $\mathrm{~J} 1000+3032$ & 3.8 & 10.3 & 23.2 & 8.3 & 0.4 & 18.0 & 12.4 & 17.8 & $8.3 \times 6.5$ & $0.4 \times 0.3$ \\
\hline 79 & KUG0959+299| & 4.3 & 11.1 & 22.7 & 10.7 & 0.7 & 17.2 & 14.8 & 17.1 & $10.7 \times 8.3$ & $0.7 \times 0.5$ \\
\hline 80 & UGC5427 & 18.8 & 37.5 & 23.2 & 38.5 & 1.8 & $|14.7|$ & 49.9 & 14.6 & $38.5 \times 24.6$ & $1.8 \times 1.2$ \\
\hline 81 & UGC5464 & 21.6 & 48.6 & 24.0 & 36.1 & 3.0 & 15.7 & 54.8 & 15.5 & $36.1 \times 16.6$ & $3.0 \times 1.4$ \\
\hline 82 & $\mathrm{~J} 1010+4617$ & 2.8 & 8.9 & 22.0 & 9.2 & 0.8 & 18.0 & 12.9 & 18.0 & $9.2 \times 4.1$ & $0.8 \times 0.4$ \\
\hline 83 & UGC5540 & 24.5 & 61.8 & 22.9 & 57.8 & 5.4 & 14.4 & 81.1 & 14.3 & $57.8 \times 20.6$ & $5.4 \times 1.9$ \\
\hline 84 & HS1013+3809 & 1.6 & 8.9 & 19.1 & 12.4 & 1.2 & 15.9 & 18.1 & 15.9 & $12.4 \times 8.1$ & $1.2 \times 0.8$ \\
\hline 85 & J1019+2923 & 6.8 & 18.4 & 23.1 & 15.0 & $\mid 1.1$ & $|17.4|$ & 22.4 & $|17.2|$ & $|15.0 \times 6.1|$ & $|1.1 \times 0.5|$ \\
\hline
\end{tabular}


Table 3. Photometric paramerets II.

\begin{tabular}{|c|c|c|c|c|c|c|c|c|}
\hline & $\begin{array}{l}\text { Name } \\
\text { (1) }\end{array}$ & $\begin{array}{c}g_{\text {tot }} \\
\text { mag } \\
(2)\end{array}$ & $\begin{array}{c}(u-g)_{\text {tot }} \\
\text { mag } \\
(3)\end{array}$ & $\begin{array}{c}(g-r)_{\text {tot }} \\
\text { mag } \\
(4)\end{array}$ & $\begin{array}{c}(r-i)_{\text {tot }} \\
\text { mag } \\
(5)\end{array}$ & $\mid \begin{array}{c}(u-g)_{\text {per }} \\
\text { mag } \\
(6)\end{array}$ & $\left|\begin{array}{c}(g-r)_{\text {per }} \\
\text { mag } \\
(7)\end{array}\right|$ & $\left|\begin{array}{c}(r-i)_{\text {per }} \\
\text { mag } \\
(8)\end{array}\right|$ \\
\hline 1 & $\mid$ HIPASSJ0626+24| & $\mid 17.53 \pm 0.02$ & $|0.88 \pm 0.03|$ & $0.29 \pm 0.01$ & $0.15 \pm 0.01$ & $0.93 \pm 0.06$ & $|0.31 \pm 0.02|$ & $|0.16 \pm 0.02|$ \\
\hline 2 & UGC3600 & $15.57 \pm 0.01$ & $1.01 \pm 0.01$ & $0.29 \pm 0.01$ & $0.12 \pm 0.01$ & $1.09 \pm 0.02$ & $0.29 \pm 0.01$ & $0.15 \pm 0.01$ \\
\hline 3 & UGC3672 & $15.72 \pm 0.01$ & $1.03 \pm 0.01$ & $0.21 \pm 0.01$ & $-0.01 \pm 0.01$ & $0.96 \pm 0.04$ & $0.21 \pm 0.02$ & $0.09 \pm 0.02$ \\
\hline 4 & $\mathrm{~J} 0713+2926$ & $16.32 \pm 0.01$ & $1.01 \pm 0.01$ & $0.27 \pm 0.01$ & $0.13 \pm 0.01$ & $1.00 \pm 0.03$ & $0.32 \pm 0.01$ & $0.15 \pm 0.02$ \\
\hline 5 & $\mathrm{~J} 0723+3621$ & $16.65 \pm 0.01$ & $1.17 \pm 0.02$ & $0.35 \pm 0.01$ & $0.18 \pm 0.01$ & $0.99 \pm 0.03$ & $0.31 \pm 0.02$ & $0.17 \pm 0.02$ \\
\hline 6 & $\mathrm{~J} 0723+3622$ & $19.24 \pm 0.02$ & $0.62 \pm 0.05$ & $-0.01 \pm 0.04$ & $0.21 \pm 0.05$ & $0.60 \pm 0.06$ & $0.01 \pm 0.05$ & $0.07 \pm 0.07$ \\
\hline 7 & $\mathrm{~J} 0723+3624$ & $21.41 \pm 0.04$ & $0.11 \pm 0.09$ & $0.08 \pm 0.07$ & $0.05 \pm 0.08$ & - & - & - \\
\hline 8 & UGC3860 & $14.89 \pm 0.01$ & $0.90 \pm 0.01$ & $0.25 \pm 0.01$ & $0.14 \pm 0.01$ & $0.95 \pm 0.02$ & $0.23 \pm 0.01$ & $0.11 \pm 0.01$ \\
\hline 9 & UGC3876 & $13.39 \pm 0.01$ & $1.11 \pm 0.01$ & $0.47 \pm 0.01$ & $0.27 \pm 0.01$ & $1.13 \pm 0.01$ & $0.38 \pm 0.01$ & $0.25 \pm 0.01$ \\
\hline 10 & J0730+4109 & $16.34 \pm 0.01$ & $0.94 \pm 0.01$ & $0.25 \pm 0.01$ & $0.15 \pm 0.01$ & $1.15 \pm 0.03$ & $0.43 \pm 0.02$ & $0.24 \pm 0.02$ \\
\hline 11 & J0737+4724 & $17.74 \pm 0.01$ & $1.00 \pm 0.03$ & $0.06 \pm 0.01$ & $0.02 \pm 0.02$ & $0.83 \pm 0.06$ & $0.09 \pm 0.03$ & $0.10 \pm 0.04$ \\
\hline 12 & UGC3966 & $15.04 \pm 0.01$ & $0.88 \pm 0.01$ & $0.12 \pm 0.01$ & $0.09 \pm 0.01$ & $0.90 \pm 0.01$ & $0.10 \pm 0.01$ & $0.09 \pm 0.01$ \\
\hline 13 & DDO47 & $14.55 \pm 0.01$ & $0.96 \pm 0.01$ & $0.31 \pm 0.01$ & $0.15 \pm 0.01$ & $1.13 \pm 0.02$ & $0.35 \pm 0.01$ & $0.18 \pm 0.01$ \\
\hline 14 & KK65 & $15.17 \pm 0.01$ & $1.08 \pm 0.01$ & $0.32 \pm 0.01$ & $0.20 \pm 0.01$ & $1.18 \pm 0.03$ & $0.43 \pm 0.01$ & $0.23 \pm 0.01$ \\
\hline 15 & $\mathrm{~J} 0744+2508$ & $17.79 \pm 0.01$ & $0.90 \pm 0.03$ & $0.25 \pm 0.01$ & $0.06 \pm 0.02$ & $1.04 \pm 0.04$ & $0.31 \pm 0.02$ & $0.16 \pm 0.02$ \\
\hline 16 & $\mathrm{~J} 0744+2506$ & $20.11 \pm 0.02$ & $1.03 \pm 0.09$ & $0.37 \pm 0.04$ & $0.15 \pm 0.05$ & $1.17 \pm 0.16$ & $0.51 \pm 0.07$ & $0.29 \pm 0.08$ \\
\hline 17 & MCG9-13-52 & $16.20 \pm 0.01$ & $0.78 \pm 0.01$ & $0.29 \pm 0.01$ & $0.16 \pm 0.01$ & $1.04 \pm 0.04$ & $0.31 \pm 0.02$ & $0.19 \pm 0.02$ \\
\hline 18 & MCG9-13-56 & $14.81 \pm 0.01$ & $0.67 \pm 0.01$ & $0.20 \pm 0.01$ & $0.05 \pm 0.01$ & $1.13 \pm 0.03$ & $0.45 \pm 0.01$ & $0.24 \pm 0.01$ \\
\hline 19 & UGC4115 & $14.49 \pm 0.01$ & $0.82 \pm 0.01$ & $0.28 \pm 0.01$ & $0.20 \pm 0.01$ & $0.90 \pm 0.03$ & $0.31 \pm 0.01$ & $0.20 \pm 0.02$ \\
\hline 20 & UGC4117 & $15.07 \pm 0.01$ & $0.89 \pm 0.01$ & $0.15 \pm 0.01$ & $0.10 \pm 0.01$ & $0.81 \pm 0.02$ & $0.05 \pm 0.01$ & $0.06 \pm 0.02$ \\
\hline 21 & UGC4148 & $15.35 \pm 0.01$ & $0.86 \pm 0.01$ & $0.21 \pm 0.01$ & $0.09 \pm 0.01$ & $0.87 \pm 0.03$ & $0.26 \pm 0.02$ & $0.02 \pm 0.02$ \\
\hline 22 & NGC2500 & $11.79 \pm 0.01$ & $0.92 \pm 0.01$ & $0.35 \pm 0.01$ & $0.23 \pm 0.01$ & $1.07 \pm 0.01$ & $0.44 \pm 0.01$ & $0.22 \pm 0.01$ \\
\hline 23 & MCG71719 & $15.13 \pm 0.01$ & $0.88 \pm 0.01$ & $0.29 \pm 0.01$ & $0.15 \pm 0.01$ & $1.10 \pm 0.02$ & $0.39 \pm 0.01$ & $0.23 \pm 0.01$ \\
\hline 24 & $\mathrm{~J} 0810+1837$ & $18.05 \pm 0.01$ & $0.89 \pm 0.04$ & $0.31 \pm 0.02$ & $0.14 \pm 0.03$ & $0.97 \pm 0.08$ & $0.40 \pm 0.04$ & $0.25 \pm 0.05$ \\
\hline 25 & J0812+4836 & $17.05 \pm 0.01$ & $0.96 \pm 0.02$ & $0.20 \pm 0.01$ & $0.06 \pm 0.01$ & $0.92 \pm 0.05$ & $0.23 \pm 0.03$ & $0.15 \pm 0.03$ \\
\hline 26 & NGC2537 & $12.11 \pm 0.01$ & $1.01 \pm 0.01$ & $0.45 \pm 0.01$ & $0.25 \pm 0.01$ & - & - & - \\
\hline 27 & IC2233 & $13.01 \pm 0.01$ & $0.90 \pm 0.01$ & $0.28 \pm 0.01$ & $0.15 \pm 0.01$ & $1.11 \pm 0.01$ & $0.31 \pm 0.01$ & $0.19 \pm 0.01$ \\
\hline 28 & NGC2541 & $11.99 \pm 0.01$ & $0.84 \pm 0.01$ & $0.34 \pm 0.01$ & $0.14 \pm 0.01$ & $0.95 \pm 0.01$ & $0.31 \pm 0.01$ & $0.15 \pm 0.01$ \\
\hline 29 & NGC2552 & $12.67 \pm 0.01$ & $0.92 \pm 0.01$ & $0.32 \pm 0.01$ & $0.15 \pm 0.01$ & $1.12 \pm 0.02$ & $0.34 \pm 0.01$ & $0.18 \pm 0.01$ \\
\hline 30 & KUG0821+321 & $16.56 \pm 0.01$ & $0.86 \pm 0.01$ & $0.33 \pm 0.01$ & $0.11 \pm 0.01$ & $1.13 \pm 0.05$ & $0.42 \pm 0.02$ & $0.26 \pm 0.02$ \\
\hline 31 & HS0822+3542 & $17.65 \pm 0.01$ & $0.65 \pm 0.02$ & $-0.03 \pm 0.02$ & $-0.37 \pm 0.03$ & - & - & - \\
\hline 32 & $\mathrm{SAO} 0822+3545$ & $17.50 \pm 0.01$ & $0.77 \pm 0.02$ & $0.00 \pm 0.01$ & $0.01 \pm 0.02$ & $0.75 \pm 0.07$ & $0.03 \pm 0.04$ & $0.04 \pm 0.05$ \\
\hline 33 & UGC4426 & $15.01 \pm 0.01$ & $1.24 \pm 0.01$ & $0.33 \pm 0.01$ & $0.27 \pm 0.01$ & $1.09 \pm 0.04$ & $0.30 \pm 0.02$ & $0.19 \pm 0.02$ \\
\hline 34 & $\mathrm{~J} 0831+4104$ & $17.37 \pm 0.01$ & $0.93 \pm 0.02$ & $0.32 \pm 0.01$ & $0.15 \pm 0.01$ & $1.15 \pm 0.05$ & $0.43 \pm 0.02$ & $0.24 \pm 0.02$ \\
\hline 35 & $\mathrm{~J} 0843+4025$ & $17.58 \pm 0.01$ & $0.98 \pm 0.03$ & $0.26 \pm 0.01$ & $0.14 \pm 0.01$ & $1.00 \pm 0.06$ & $0.30 \pm 0.03$ & $0.16 \pm 0.03$ \\
\hline 36 & J0845+1519 & $18.25 \pm 0.01$ & $1.21 \pm 0.04$ & $0.41 \pm 0.02$ & $0.25 \pm 0.02$ & $0.92 \pm 0.08$ & $0.38 \pm 0.03$ & $0.25 \pm 0.03$ \\
\hline 37 & J0852+1350 & $17.06 \pm 0.01$ & $0.85 \pm 0.02$ & $0.32 \pm 0.01$ & $0.14 \pm 0.01$ & $1.11 \pm 0.07$ & $0.42 \pm 0.03$ & $0.22 \pm 0.03$ \\
\hline 38 & J0852+1351 & $19.48 \pm 0.02$ & $0.79 \pm 0.06$ & $0.33 \pm 0.03$ & $0.02 \pm 0.03$ & $0.91 \pm 0.11$ & $0.33 \pm 0.05$ & $0.20 \pm 0.06$ \\
\hline 39 & UGC4704 & $14.50 \pm 0.01$ & $0.89 \pm 0.01$ & $0.27 \pm 0.01$ & $0.14 \pm 0.01$ & $1.03 \pm 0.01$ & $0.35 \pm 0.01$ & $0.24 \pm 0.01$ \\
\hline 40 & J0859+3923 & $16.91 \pm 0.01$ & $1.10 \pm 0.02$ & $0.32 \pm 0.01$ & $0.18 \pm 0.01$ & $1.16 \pm 0.05$ & $0.42 \pm 0.02$ & $0.25 \pm 0.03$ \\
\hline 41 & J0900+3222 & $18.60 \pm 0.01$ & $1.20 \pm 0.04$ & $0.43 \pm 0.02$ & $0.20 \pm 0.02$ & $1.13 \pm 0.07$ & $0.43 \pm 0.03$ & $0.22 \pm 0.03$ \\
\hline 42 & UGC4722 & $14.56 \pm 0.01$ & $0.92 \pm 0.01$ & $0.19 \pm 0.01$ & $0.07 \pm 0.01$ & $1.10 \pm 0.03$ & $0.33 \pm 0.01$ & $0.18 \pm 0.02$ \\
\hline 43 & J0900+4908 & $18.17 \pm 0.01$ & $1.18 \pm 0.05$ & $0.29 \pm 0.02$ & $0.21 \pm 0.03$ & $0.97 \pm 0.05$ & $0.38 \pm 0.03$ & $0.19 \pm 0.03$ \\
\hline 44 & J0908+0657 & $16.70 \pm 0.01$ & $1.16 \pm 0.02$ & $0.37 \pm 0.01$ & $0.16 \pm 0.01$ & $1.10 \pm 0.04$ & $0.44 \pm 0.02$ & $0.25 \pm 0.02$ \\
\hline 45 & J0908+0517 & $16.90 \pm 0.01$ & $0.74 \pm 0.02$ & $0.22 \pm 0.01$ & $-0.13 \pm 0.01$ & $1.03 \pm 0.06$ & $0.24 \pm 0.03$ & $0.11 \pm 0.03$ \\
\hline 46 & J0911+3135 & $17.76 \pm 0.01$ & $0.62 \pm 0.02$ & $0.18 \pm 0.02$ & $0.06 \pm 0.02$ & $1.02 \pm 0.06$ & $0.28 \pm 0.03$ & $0.18 \pm 0.03$ \\
\hline 47 & IC2450 & $13.67 \pm 0.01$ & $1.27 \pm 0.01$ & $0.48 \pm 0.01$ & $0.23 \pm 0.01$ & $1.43 \pm 0.02$ & $0.55 \pm 0.01$ & $0.32 \pm 0.01$ \\
\hline 48 & $\mathrm{~J} 0926+3343$ & $17.05 \pm 0.01$ & $0.81 \pm 0.02$ & $0.07 \pm 0.01$ & $-0.08 \pm 0.02$ & $0.98 \pm 0.06$ & $0.14 \pm 0.03$ & $0.07 \pm 0.04$ \\
\hline 49 & J0928+2845 & $16.39 \pm 0.01$ & $1.13 \pm 0.02$ & $0.45 \pm 0.01$ & $0.24 \pm 0.01$ & $1.31 \pm 0.04$ & $0.55+001$ & $0.25 \pm 0.01 \mid$ \\
\hline
\end{tabular}


Table 3. Photometric paramerets II. (Condt.)

\begin{tabular}{|c|c|c|c|c|c|c|c|c|}
\hline & $\begin{array}{c}\text { Name } \\
\text { (1) }\end{array}$ & $\begin{array}{c}g_{\text {tot }} \\
\text { mag } \\
(2)\end{array}$ & $\mid \begin{array}{c}(u-g)_{\text {tot }} \\
\text { mag } \\
(3)\end{array}$ & $\begin{array}{c}(g-r)_{\text {tot }} \\
\text { mag } \\
(4)\end{array}$ & $\begin{array}{c}(r-i)_{\text {tot }} \\
\text { mag } \\
(5)\end{array}$ & $\begin{array}{c}(u-g)_{\text {per }} \\
\text { mag } \\
(6)\end{array}$ & $\mid \begin{array}{c}(g-r)_{\text {per }} \\
\text { mag } \\
(7)\end{array}$ & $\mid \begin{array}{c}(r-i)_{\text {per }} \\
\text { mag } \\
(8)\end{array}$ \\
\hline 50 & J0929+2502 & $\mid 18.95 \pm 0.02$ & $0.87 \pm 0.05$ & $0.16 \pm 0.03$ & $0.11 \pm 0.04$ & $0.94 \pm 0$. & $0.19 \pm$ & $0.13 \pm 0.06$ \\
\hline 51 & J0929+1155 & $16.89 \pm 0.01$ & $0.95 \pm 0.02$ & $0.22 \pm 0.01$ & $0.15 \pm 0.01$ & $1.04 \pm 0.08$ & $0.35 \pm 0.04$ & $0.20 \pm 0.04$ \\
\hline 52 & J0931+2717 & $17.70 \pm 0.01$ & $1.17 \pm 0.04$ & $0.22 \pm 0.02$ & $0.11 \pm 0.02$ & $1.04 \pm 0.08$ & $0.26 \pm 0.01$ & $0.15 \pm 0.05$ \\
\hline 53 & CGCG035-007 & $15.05 \pm 0.01$ & $1.13 \pm 0.01$ & $0.42 \pm 0.01$ & $0.20 \pm 0.01$ & $1.29 \pm 0.02$ & $0.51 \pm 0.01$ & $0.22 \pm 0.01$ \\
\hline 54 & KUG0934+277 & $16.17 \pm 0.01$ & $0.99 \pm 0.01$ & $0.30 \pm 0.01$ & $0.15 \pm 0.01$ & $0.96 \pm 0.04$ & $0.37 \pm 0.02$ & $0.20 \pm 0.02$ \\
\hline 55 & J0940+4459 & $17.67 \pm 0.01$ & $0.97 \pm 0.03$ & $0.34 \pm 0.01$ & $0.19 \pm 0.02$ & $1.15 \pm 0.07$ & $0.40 \pm 0.03$ & $0.20 \pm 0.04$ \\
\hline 56 & KISSB23 & $15.77 \pm 0.01$ & $0.75 \pm 0.01$ & $0.20 \pm 0.01$ & $0.16 \pm 0.01$ & $1.08 \pm 0.03$ & $0.38 \pm 0.01$ & $0.23 \pm 0.02$ \\
\hline 57 & J0942+0937 & $17.80 \pm 0.03$ & $1.02 \pm 0.03$ & $0.37 \pm 0.01$ & $0.26 \pm 0.01$ & $0.94 \pm 0.07$ & $0.38 \pm 0.04$ & $0.24 \pm 0.04$ \\
\hline 58 & UGC5186 & $15.68 \pm 0.01$ & $1.09 \pm 0.01$ & $0.27 \pm 0.01$ & $0.18 \pm 0.01$ & $1.12 \pm 0.03$ & $0.39 \pm 0.01$ & $0.22 \pm 0.01$ \\
\hline 59 & J0943+4134 & $17.30 \pm 0.01$ & $1.10 \pm 0.02$ & $0.34 \pm 0.01$ & $0.16 \pm 0.01$ & $1.06 \pm 0.05$ & $0.40 \pm 0.03$ & $0.21 \pm 0.03$ \\
\hline 60 & J0944+1000 & $16.62 \pm 0.01$ & $0.91 \pm 0.01$ & $0.32 \pm 0.01$ & $0.13 \pm 0.01$ & $0.93 \pm 0.04$ & $0.34 \pm 0.02$ & $0.24 \pm 0.02$ \\
\hline 61 & IC559 & $14.43 \pm 0.01$ & $1.02 \pm 0.01$ & $0.33 \pm 0.01$ & $0.18 \pm 0.01$ & $1.10 \pm 0.02$ & $0.43 \pm 0.01$ & $0.26 \pm 0.01$ \\
\hline 62 & UGC5209 & $15.98 \pm 0.01$ & $0.95 \pm 0.01$ & $0.33 \pm 0.01$ & $0.18 \pm 0.01$ & $0.95 \pm 0.03$ & $0.37 \pm 0.02$ & $0.21 \pm 0.02$ \\
\hline 63 & J0947+4138 & $17.65 \pm 0.01$ & $0.87 \pm 0.03$ & $0.12 \pm 0.02$ & $-0.03 \pm 0.03$ & $0.93 \pm 0.06$ & $0.28 \pm 0.03$ & $0.17 \pm 0.04$ \\
\hline 64 & MRK407 & $14.96 \pm 0.01$ & $0.86 \pm 0.01$ & $0.28 \pm 0.01$ & $0.10 \pm 0.01$ & - & - & - \\
\hline 65 & J0947+3908 & $16.54 \pm 0.01$ & $0.91 \pm 0.01$ & $0.23 \pm 0.01$ & $0.08 \pm 0.01$ & $0.95 \pm 0.03$ & $0.27 \pm 0.02$ & $0.14 \pm 0.02$ \\
\hline 66 & J0947+3905 & $17.71 \pm 0.01$ & $1.21 \pm 0.03$ & $0.27 \pm 0.01$ & $0.15 \pm 0.02$ & $1.16 \pm 0.06$ & $0.41 \pm 0.03$ & $0.21 \pm 0.03$ \\
\hline 67 & UGC5272b & $17.29 \pm 0.01$ & $0.92 \pm 0.02$ & $0.12 \pm 0.01$ & $0.08 \pm 0.02$ & $0.89 \pm 0.06$ & $0.18 \pm 0.03$ & $0.11 \pm 0.04$ \\
\hline 68 & UGC5272 & $14.18 \pm 0.01$ & $0.67 \pm 0.01$ & $0.13 \pm 0.01$ & $0.07 \pm 0.01$ & $0.87 \pm 0.03$ & $0.17 \pm 0.02$ & $0.15 \pm 0.02$ \\
\hline 69 & UGC5288 & $14.08 \pm 0.01$ & $0.89 \pm 0.01$ & $0.34 \pm 0.01$ & $0.19 \pm 0.01$ & $1.02 \pm 0.02$ & $0.41 \pm 0.01$ & $0.27 \pm 0.01$ \\
\hline 70 & J0951+3842 & $17.16 \pm 0.01$ & $0.93 \pm 0.02$ & $0.21 \pm 0.01$ & $0.14 \pm 0.01$ & $0.98 \pm 0.05$ & $0.37 \pm 0.03$ & $0.19 \pm 0.03$ \\
\hline 71 & J0954+3620 & $17.75 \pm 0.01$ & $1.10 \pm 0.03$ & $0.21 \pm 0.02$ & $0.08 \pm 0.02$ & $1.11 \pm 0.07$ & $0.28 \pm 0.04$ & $0.19 \pm 0.05$ \\
\hline 72 & IC 2520 & $13.85 \pm 0.01$ & $1.05 \pm 0.01$ & $0.59 \pm 0.01$ & $0.25 \pm 0.01$ & $0.98 \pm 0.04$ & $0.43 \pm 0.02$ & $0.27 \pm 0.02$ \\
\hline 73 & J0956+2716 & $17.81 \pm 0.01$ & $0.87 \pm 0.03$ & $0.28 \pm 0.02$ & $0.15 \pm 0.02$ & $1.05 \pm 0.08$ & $0.32 \pm 0.04$ & $0.20 \pm 0.04$ \\
\hline 74 & UGC5340 & $14.46 \pm 0.01$ & $0.64 \pm 0.01$ & $0.03 \pm 0.01$ & $0.03 \pm 0.01$ & $0.77 \pm 0.01$ & $0.06 \pm 0.01$ & $0.07 \pm 0.01$ \\
\hline 75 & J0957+2745 & $17.85 \pm 0.01$ & $1.04 \pm 0.03$ & $0.24 \pm 0.01$ & $0.10 \pm 0.01$ & $0.93 \pm 0.07$ & $0.33 \pm 0.03$ & $0.14 \pm 0.04$ \\
\hline 76 & UGC5354 & $13.84 \pm 0.01$ & $0.94 \pm 0.01$ & $0.27 \pm 0.01$ & $0.14 \pm 0.01$ & $1.03 \pm 0.02$ & $0.24 \pm 0.01$ & $0.13 \pm 0.01$ \\
\hline 77 & $\mathrm{PC} 0956+4751$ & $16.72 \pm 0.01$ & $0.84 \pm 0.01$ & $0.31 \pm 0.01$ & $0.07 \pm 0.01$ & $0.98 \pm 0.04$ & $0.34 \pm 0.02$ & $0.18 \pm 0.03$ \\
\hline 78 & $\mathrm{~J} 1000+3032$ & $17.78 \pm 0.01$ & $1.14 \pm 0.03$ & $0.40 \pm 0.01$ & $0.12 \pm 0.01$ & $1.06 \pm 0.09$ & $0.39 \pm 0.05$ & $0.17 \pm 0.05$ \\
\hline 79 & KUG0959+299 & $17.05 \pm 0.01$ & $0.95 \pm 0.02$ & $0.23 \pm 0.01$ & $0.07 \pm 0.01$ & $1.10 \pm 0.06$ & $0.37 \pm 0.03$ & $0.20 \pm 0.03$ \\
\hline 80 & UGC5427 & $14.58 \pm 0.01$ & $0.94 \pm 0.01$ & $0.29 \pm 0.01$ & $0.18 \pm 0.01$ & $1.09 \pm 0.02$ & $0.33 \pm 0.01$ & $0.21 \pm 0.01$ \\
\hline 81 & UGC5464 & $15.42 \pm 0.01$ & $0.91 \pm 0.01$ & $0.38 \pm 0.01$ & $0.18 \pm 0.01$ & $1.06 \pm 0.03$ & $0.43 \pm 0.01$ & $0.25 \pm 0.01$ \\
\hline 82 & $\mathrm{~J} 1010+4617$ & $17.92 \pm 0.01$ & $0.78 \pm 0.03$ & $0.25 \pm 0.02$ & $0.11 \pm 0.02$ & $0.86 \pm 0.08$ & $0.31 \pm 0.04$ & $0.20 \pm 0.04$ \\
\hline 83 & UGC5540 & $14.30 \pm 0.01$ & $0.98 \pm 0.00$ & $0.32 \pm 0.01$ & $0.17 \pm 0.01$ & $1.09 \pm 0.02$ & $0.42 \pm 0.01$ & $0.26 \pm 0.01$ \\
\hline 84 & HS1013+3809 & $15.82 \pm 0.01$ & $0.59 \pm 0.02$ & $-0.11 \pm 0.02$ & $-0.31 \pm 0.04$ & - & - & - \\
\hline 85 & $\mathrm{~J} 1019+2923$ & $17.17 \pm 0.01$ & $1.12 \pm 0.02$ & $0.23 \pm 0.01$ & $0.03 \pm 0.01$ & $1.08 \pm 0.05$ & $0.27 \pm 0.02$ & $0.13 \pm 0.03$ \\
\hline
\end{tabular}


Table 4. Photometric paramerets III.

\begin{tabular}{|c|c|c|c|c|c|c|c|}
\hline & $\begin{array}{c}\text { Name } \\
\text { (1) }\end{array}$ & $\begin{array}{c}\mu_{0}(g) \\
m / \square^{\prime \prime} \\
(2)\end{array}$ & $\begin{array}{c}\mu_{0}(r) \\
m / \square^{\prime \prime} \\
(3)\end{array}$ & $\left|\begin{array}{c}\mu_{0}(B) \\
m / \square^{\prime \prime} \\
(4)\end{array}\right|$ & $\mid \begin{array}{c}\mu_{0, c, \mathrm{i}}(B) \\
m / \square^{\prime \prime} \\
(5)\end{array}$ & $\begin{array}{c}\alpha(g) \\
\prime \prime \\
(6)\end{array}$ & $\begin{array}{c}n(g) \\
\text { Sersic } \\
(7)\end{array}$ \\
\hline 1 & $\mid$ HIPASSJ0626+24| & $23.54 \pm 0.06$ & $\mid 22.79 \pm 0.05$ & 24.00 & 22.83 & $8.68 \pm 0.38$ & $1.64 \pm 0.10$ \\
\hline 2 & UGC3600 & $22.92 \pm 0.08$ & $22.55 \pm 0.07$ & 23.26 & 24.31 & $11.57 \pm 0.35$ & $1.00 \pm 0.01$ \\
\hline 3 & |UGC3672 & $23.13 \pm 0.25$ & $23.20 \pm 0.19$ & 23.34 & 23.72 & $13.22 \pm 2.42$ & $1.19 \pm 0.15$ \\
\hline 4 & J0713+2926 & $22.49 \pm 0.04$ & $22.09 \pm 0.05$ & 22.84 & 23.21 & $8.15 \pm 0.28$ & $1.26 \pm 0.03$ \\
\hline 5 & J0723+3621 & $22.58 \pm 0.04$ & $22.16 \pm 0.07$ & 22.94 & 24.15 & $6.69 \pm 0.21$ & $1.10 \pm 0.02$ \\
\hline 6 & J0723+3622 & $24.13 \pm 0.20$ & $24.14 \pm 0.14$ & 24.35 & 24.60 & $5.44 \pm 0.67$ & $2.19 \pm 0.57$ \\
\hline 7 & J0723+3624 & $24.29 \pm 0.16$ & $24.14 \pm 0.23$ & 24.56 & 25.77 & $2.11 \pm 0.22$ & $1.00 \pm 0.01$ \\
\hline 8 & UGC3860 & $22.99 \pm 0.06$ & $22.60 \pm 0.05$ & 23.34 & 24.00 & $22.42 \pm 0.86$ & $1.79 \pm 0.10$ \\
\hline 9 & UGC3876 & $22.01 \pm 0.05$ & $21.27 \pm 0.06$ & 22.47 & 22.91 & $27.16 \pm 0.99$ & $1.49 \pm 0.05$ \\
\hline 10 & J0730+4109 & $21.08 \pm 0.16$ & $20.57 \pm 0.15$ & 21.47 & 22.00 & $3.30 \pm 0.14$ & $1.00 \pm 0.01$ \\
\hline 11 & J0737+4724 & $22.93 \pm 0.12$ & $22.78 \pm 0.12$ & 23.20 & 24.17 & $5.13 \pm 0.48$ & $1.27 \pm 0.10$ \\
\hline 12 & UGC3966 & $23.71 \pm 0.11$ & $23.30 \pm 0.14$ & 24.07 & 24.04 & $30.19 \pm 1.95$ & $1.91 \pm 0.21$ \\
\hline 13 & DDO47 & $23.18 \pm 0.05$ & $23.05 \pm 0.03$ & 23.45 & 24.30 & $22.24 \pm 1.14$ & $1.01 \pm 0.03$ \\
\hline 14 & KK65 & $21.97 \pm 0.08$ & $21.70 \pm 0.06$ & 22.28 & 23.76 & $9.74 \pm 0.64$ & $1.07 \pm 0.04$ \\
\hline 15 & J0744+2508 & $22.12 \pm 0.13$ & $21.85 \pm 0.05$ & 22.43 & 22.48 & $3.92 \pm 0.34$ & $1.66 \pm 0.16$ \\
\hline 16 & J0744+2506 & $22.90 \pm 0.07$ & $22.79 \pm 0.06$ & 23.16 & 23.23 & $1.81 \pm 0.09$ & $1.37 \pm 0.07$ \\
\hline 17 & MCG9-13-52 & $23.41 \pm 0.06$ & $23.06 \pm 0.09$ & 23.75 & 23.90 & $15.11 \pm 0.59$ & $1.68 \pm 0.07$ \\
\hline 18 & MCG9-13-56 & $20.29 \pm 0.08$ & $20.19 \pm 0.06$ & 20.55 & 20.69 & $5.30 \pm 0.35$ & $1.08 \pm 0.04$ \\
\hline 19 & UGC4115 & $22.13 \pm 0.17$ & $21.97 \pm 0.16$ & 22.41 & 22.89 & $16.36 \pm 2.27$ & $1.16 \pm 0.14$ \\
\hline 20 & UGC4117 & $22.02 \pm 0.03$ & $21.78 \pm 0.04$ & 22.32 & 22.46 & $11.43 \pm 0.30$ & $1.21 \pm 0.02$ \\
\hline 21 & UGC4148 & $23.42 \pm 0.09$ & $23.14 \pm 0.10$ & 23.73 & 24.61 & $14.42 \pm 0.46$ & $1.00 \pm 0.01$ \\
\hline 22 & NGC2500 & $21.20 \pm 0.05$ & $21.24 \pm 0.04$ & 21.41 & 21.25 & $39.00 \pm 1.16$ & $1.43 \pm 0.03$ \\
\hline 23 & MCG71719 & 22.17 & $22.02 \pm 0.13$ & 22.44 & 22.83 & $10.54 \pm 1.18$ & $1.15 \pm 0.08$ \\
\hline 24 & $\mathrm{~J} 0810+1837$ & $22.44 \pm 0.11$ & $22.20 \pm 0.07$ & 22.74 & 24.10 & $3.10 \pm 0.30$ & $1.01 \pm 0.06$ \\
\hline 25 & $\mathrm{~J} 0812+4836$ & $22.06 \pm 0.08$ & $21.74 \pm 0.05$ & 22.39 & 23.27 & $5.02 \pm 0.29$ & $1.39 \pm 0.07$ \\
\hline 26 & NGC2537 & $20.56 \pm 0.08$ & $19.89 \pm 0.07$ & 21.00 & 20.83 & $15.10 \pm 0.36$ & $1.00 \pm 0.01$ \\
\hline 27 & |IC2233 & $22.29 \pm 0.12$ & $21.74 \pm 0.10$ & 22.69 & 23.60 & $27.54 \pm 1.19$ & $1.00 \pm 0.01$ \\
\hline 28 & NGC2541 & $22.18 \pm 0.06$ & $21.60 \pm 0.06$ & 22.59 & 22.97 & $52.15 \pm 2.42$ & $1.34 \pm 0.05$ \\
\hline 29 & NGC2552 & $22.59 \pm 0.02$ & $22.12 \pm 0.03$ & 22.96 & 23.33 & $53.37 \pm 0.77$ & $1.96 \pm 0.04$ \\
\hline 30 & KUG0821 & $21.80 \pm 0.10$ & $21.54 \pm 0.10$ & 22.11 & 2.49 & $5.30 \pm 0.41$ & $1.27 \pm 0.08$ \\
\hline 31 & HSO $0822+354$ & $21.92 \pm 0.14$ & $20.80 \pm 0.12$ & 22.50 & 2.65 & $2.01 \pm 0.08$ & $1.00 \pm 0.01$ \\
\hline 32 & $\mathrm{SAO} 0822+3$ & $22.96 \pm 0.05$ & $22.90 \pm 0.09$ & 23.21 & 3.30 & $6.60 \pm 0.21$ & $1.62 \pm 0.06$ \\
\hline 33 & UGC4426 & $23.62 \pm 0.05$ & $23.16 \pm 0.04$ & 23.99 & 24.37 & $25.95 \pm 1.18$ & $1.30 \pm 0.06$ \\
\hline 34 & $\mathrm{~J} 0831+4104$ & $21.65 \pm 0.15$ & $21.42 \pm 0.14$ & 21.95 & 21.93 & $2.99 \pm 0.41$ & $1.08 \pm 0.10$ \\
\hline 35 & $\mathrm{~J} 0843+4025$ & $21.62 \pm 0.20$ & $21.49 \pm 0.07$ & 21.89 & 22.25 & $2.84 \pm 0.48$ & $1.14 \pm 0.14$ \\
\hline 36 & $\mathrm{~J} 0845+1519$ & $22.37 \pm 0.19$ & $22.07 \pm 0.19$ & 22.69 & 22.92 & $2.77 \pm 0.47$ & $1.05 \pm 0.11$ \\
\hline 37 & $\mathrm{~J} 0852+1350$ & $23.02 \pm 0.11$ & $22.80 \pm 0.08$ & 23.32 & 23.53 & $7.76 \pm 0.63$ & $1.39 \pm 0.10$ \\
\hline 38 & J0852+1351 & 22.1 & 22.41 & 22.27 & 22.76 & $1.31 \pm$ & $1.00 \pm 0.01$ \\
\hline 39 & UGC4704 & 23.99 & $23.49 \pm$ & 24.37 & 24.50 & $32.01 \pm$ & $1.10 \pm 0.09$ \\
\hline 40 & $\mathrm{~J} 0859+$ & 23.04 & $22.21 \exists$ & 23.53 & 23.52 & $7.20 \pm 2.02$ & $1.32 \pm 0.27$ \\
\hline 41 & J0900+3222 & $22.76 \pm 0.09$ & $22.44 \pm 0.07$ & 23.09 & 23.60 & $3.09 \pm 0.24$ & $1.18 \pm 0.07$ \\
\hline 42 & UGC4722 & $22.2 ?$ & $22.35 \pm 0.27$ & 22.50 & 23.46 & $12.66 \pm 1.49$ & $1.02 \pm 0.06$ \\
\hline 43 & J0900+4908 & $23.27 \pm 0.05$ & $22.75 \pm 0.11$ & 23.66 & 24.04 & $4.90 \pm 0.20$ & $1.24 \pm 0.05$ \\
\hline 44 & J0908+0657 & $21.78 \pm 0.14$ & $21.46 \pm 0.07$ & 22.11 & 22.58 & $4.09 \pm 0.43$ & $1.00 \pm 0.05$ \\
\hline 45 & J0908+0517 & $23.46 \pm 0.11$ & $23.28 \pm 0.10$ & 23.74 & 23.56 & $8.50 \pm 0.91$ & $1.11 \pm 0.09$ \\
\hline 46 & J0911+3135 & $21.52 \pm 0.20$ & $21.37 \pm 0.18$ & 21.79 & 21.91 & $2.78 \pm 0.40$ & $1.39 \pm 0.16$ \\
\hline 47 & IC2450 & $20.73 \pm 0.05$ & $20.17 \pm 0.04$ & 21.13 & 21.58 & $9.00 \pm 0.15$ & $1.00 \pm 0.01$ \\
\hline 48 & J0926+3343 & $23.32 \pm 0.07$ & $23.26 \pm 0.07$ & 23.57 & 24.82 & $7.29 \pm 0.50$ & $1.04 \pm 0.06$ \\
\hline 49 & J0928+2845 & $21.01 \pm 0.16$ & $20.67 \pm 0.14$ & 21.34 & 22.10 & $3.65 \pm 0.54$ & $1.07 \pm 0.09$ \\
\hline 50 & J0929+2502 & $24.10 \pm 0.18$ & $23.98 \pm 0.15$ & $\mid 24.36$ & 24.38 & $5.61 \pm 0.82$ & $1.38 \pm 0.23$ \\
\hline
\end{tabular}


Table 4. Photometric paramerets III. (Condt.)

\begin{tabular}{|c|c|c|c|c|c|c|c|}
\hline & $\begin{array}{c}\text { Name } \\
(1)\end{array}$ & $\begin{array}{c}\mu_{0}(g) \\
m / \square^{\prime \prime} \\
(2)\end{array}$ & $\begin{array}{c}\mu_{0}(r) \\
m / \square^{\prime \prime} \\
(3)\end{array}$ & $\left|\begin{array}{c}\mu_{0}(B) \\
m / \square^{\prime \prime} \\
(4)\end{array}\right|$ & $\mid \begin{array}{c}\mu_{0, \mathrm{c}, \mathrm{i}}(B) \\
m / \square^{\prime \prime} \\
(5)\end{array}$ & $\begin{array}{c}\alpha(g) \\
\prime \prime \\
(6)\end{array}$ & $\begin{array}{c}n(g) \\
\text { Sersic } \\
(7)\end{array}$ \\
\hline 51 & J0929+1155 & $\mid 22.88 \pm 0.35$ & $\mid 22.77 \pm 0.10$ & 23.14 & 24.15 & $6.73 \pm 1.73$ & $\mid 1.12 \pm 0.19$ \\
\hline 52 & $\mathrm{~J} 0931+2717$ & $22.58 \pm 0.47$ & $22.81 \pm 0.43$ & 22.74 & 23.75 & $3.91 \pm 1.27$ & $1.07 \pm 0.19$ \\
\hline 53 & CGCG035-007 & $20.79 \pm 0.09$ & $20.47 \pm 0.09$ & 21.12 & 21.29 & $5.73 \pm 0.45$ & $1.02 \pm 0.04$ \\
\hline 54 & KUG0934+277 & $22.52 \pm 0.05$ & $22.26 \pm 0.04$ & 22.83 & 23.09 & $8.91 \pm 0.38$ & $1.25 \pm 0.04$ \\
\hline 55 & J0940+4459 & $21.23 \pm 0.14$ & $20.91 \pm 0.12$ & 21.56 & 21.96 & $2.24 \pm 0.25$ & $1.14 \pm 0.08$ \\
\hline 56 & |KISSB23 & $22.28 \pm 0.36$ & $22.03 \pm 0.34$ & 22.59 & 23.82 & $6.71 \pm 1.53$ & $1.02 \pm 0.11$ \\
\hline 57 & J0942+0937 & $23.70 \pm 0.09$ & $23.16 \pm 0.08$ & 24.10 & 24.27 & $7.79 \pm 0.53$ & $1.43 \pm 0.10$ \\
\hline 58 & UGC5186 & $22.71 \pm 0.09$ & $22.37 \pm 0.11$ & 23.04 & 24.88 & $9.03 \pm 0.24$ & $1.00 \pm 0.01$ \\
\hline 59 & J0943+4134 & $21.68 \pm 0.06$ & $21.41 \pm 0.05$ & 21.99 & 22.84 & $2.97 \pm 0.08$ & $1.00 \pm 0.01$ \\
\hline 60 & J0944+1000 & $23.42 \pm 0.02$ & $23.07 \pm 0.04$ & 23.76 & 23.76 & $12.55 \pm 0.19$ & $1.75 \pm 0.04$ \\
\hline 61 & IC559 & $22.62 \pm 0.43$ & $22.12 \pm 0.46$ & 23.00 & 23.04 & $16.82 \pm 4.84$ & $1.11 \pm 0.18$ \\
\hline 62 & UGC5209 & $23.28 \pm 0.06$ & $22.99 \pm 0.04$ & 23.60 & 23.91 & $15.41 \pm 0.60$ & $1.61 \pm 0.07$ \\
\hline 63 & J0947+4138 & $22.45 \pm 0.21$ & $22.18 \pm 0.24$ & 22.76 & 23.72 & $2.78 \pm 0.18$ & $1.00 \pm 0.01$ \\
\hline 64 & MRK407 & $20.71 \pm 0.69$ & $20.77 \pm 1.13$ & 20.92 & 21.20 & $6.58 \pm 2.39$ & $1.17 \pm 0.22$ \\
\hline 65 & J0947+3908 & $22.45 \pm 0.04$ & $22.19 \pm 0.04$ & 22.76 & 23.23 & $8.50 \pm 0.22$ & $1.95 \pm 0.07$ \\
\hline 66 & J0947+3905 & $23.03 \pm 0.21$ & $22.74 \pm 0.17$ & 23.35 & 23.95 & $3.82 \pm 0.33$ & $1.00 \pm 0.01$ \\
\hline 67 & UGC5272b & $22.09 \pm 0.12$ & $22.05 \pm 0.08$ & 22.33 & 22.82 & $4.23 \pm 0.41$ & $1.23 \pm 0.09$ \\
\hline 68 & UGC5272 & $21.76 \pm 0.05$ & $21.58 \pm 0.03$ & 22.04 & 23.00 & $12.84 \pm 0.26$ & $1.00 \pm 0.01$ \\
\hline 69 & UGC5288 & $21.88 \pm 0.40$ & $21.92 \pm 0.25$ & 22.09 & 22.04 & $3.44 \pm 1.02$ & $1.02 \pm 0.13$ \\
\hline 70 & J0951+3842 & $22.47 \pm 0.06$ & $22.39 \pm 0.03$ & 22.72 & 22.79 & $5.13 \pm 0.26$ & $1.16 \pm 0.04$ \\
\hline 71 & J0954+3620 & $22.15 \pm 0.08$ & $22.16 \pm 0.06$ & 22.37 & 23.04 & $3.23 \pm 0.22$ & $1.10 \pm 0.05$ \\
\hline 72 & IC2520 & $19.69 \pm 0.07$ & $18.72 \pm 0.07$ & 20.22 & 20.29 & $7.26 \pm 0.36$ & $1.32 \pm 0.05$ \\
\hline 73 & J0956+2716 & $23.64 \pm 0.12$ & $23.37 \pm 0.13$ & 23.95 & 24.88 & $6.21 \pm 0.74$ & $1.04 \pm 0.09$ \\
\hline 74 & UGC5340 & $21.99 \pm 0.04$ & $21.87 \pm 0.04$ & 22.25 & 23.16 & $11.63 \pm 0.20$ & $1.00 \pm 0.01$ \\
\hline 75 & $\mathrm{~J} 0957+2745$ & $21.68 \pm 0.12$ & $21.54 \pm 0.16$ & 21.95 & 22.64 & $2.47 \pm 0.25$ & $1.05 \pm 0.06$ \\
\hline 76 & UGC5354 & $21.42 \pm 0.06$ & $20.87 \pm 0.06$ & 21.82 & 22.42 & $13.98 \pm 0.78$ & $1.11 \pm 0.04$ \\
\hline 77 & $\mathrm{PC} 0956+4751$ & $22.33 \pm 0.24$ & $21.93 \pm 0.06$ & 22.68 & 24.14 & $5.19 \pm 0.84$ & $1.03 \pm 0.08$ \\
\hline 78 & $\mathrm{~J} 1000+3032$ & $22.17 \pm 0.13$ & $22.08 \pm 0.18$ & 22.43 & 22.67 & $3.38 \pm 0.35$ & $1.16 \pm 0.08$ \\
\hline 79 & KUG0959+299 & $21.56 \pm 0.12$ & $21.49 \pm 0.11$ & 21.81 & 22.07 & $3.5 \pm 0.34$ & $1.13 \pm 0.07$ \\
\hline 80 & UGC5427 & $21.92 \pm 0.04$ & $21.56 \pm 0.03$ & 22.26 & 22.68 & $14.97 \pm 0.41$ & $1.44 \pm 0.03$ \\
\hline 81 & UGC5464 & $22.62 \pm 0.05$ & $22.26 \pm 0.07$ & 22.96 & 23.79 & $12.79 \pm 0.44$ & $1.23 \pm 0.03$ \\
\hline 82 & $\mathrm{~J} 1010+4617$ & $21.25 \pm 0.14$ & $21.18 \pm 0.04$ & 21.50 & 23.00 & $1.85 \pm 0.24$ & $1.03 \pm 0.08$ \\
\hline 83 & UGC5540 & $21.63 \pm 0.13$ & $21.23 \pm 0.23$ & 21.98 & 23.25 & $12.03 \pm 1.13$ & $1.09 \pm 0.06$ \\
\hline 84 & HS1013+3809 & $21.44 \pm 0.56$ & $21.66 \pm 0.85$ & 21.60 & 22.02 & $2.98 \pm 1.02$ & $0.97 \pm 0.14$ \\
\hline 85 & $\mathrm{~J} 1019+2923$ & $21.79 \pm 0.06$ & $21.52 \pm 0.05$ & 22.10 & 23.77 & $3.34 \pm 0.08$ & $1.00 \pm 0.01$ \\
\hline
\end{tabular}

